\title{
A large scale CO survey of the Galactic center region
}

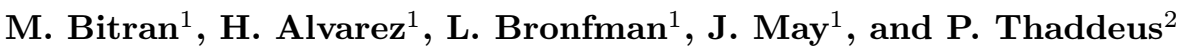 \\ 1 Departamento de Astronomía, Universidad de Chile, Casilla 36-D, Santiago, Chile \\ 2 Harvard-Smithonian Center for Astrophysics, 60 Garden Street, Cambridge, MA 02138, U.S.A.
}

Received October 21, 1996; accepted January 2, 1997

\begin{abstract}
We present the data from a $\mathrm{CO}(J=1 \rightarrow$ 0 ) survey of the central region of our Galaxy. The observations were obtained with the 1.2-m Southern Millimeter-wave Telescope at Cerro Tololo Interamerican Observatory, Chile. This instrument has a full beam-width at half maximum (FWHM) of $8.8^{\prime}$ at $115 \mathrm{GHz}$, the frequency of the observed transition. The survey covers a strip $4^{\circ}$ wide in latitude along the galactic equator in the range $-12^{\circ} \leq l \leq+13^{\circ}$. The spatial sampling interval was $7.5^{\prime}$ ( 0.85 beam-widths) for $|b| \leq 1^{\circ}$, and $15^{\prime}$ (1.7 beamwidths) for $1^{\circ}<|b| \leq 2^{\circ}$. The velocity coverage allowed by the filterbank was of $333 \mathrm{~km} \mathrm{~s}^{-1}$ with a resolution of $1.3 \mathrm{~km} \mathrm{~s}^{-1}$ at $115 \mathrm{GHz}$. For the central few degrees $\left(-4.5^{\circ}<l \leq 5^{\circ}\right)$ where this velocity span was not wide enough, two spectra, taken with different velocities with respect to the local standard of rest (VLSR), were combined in order to cover the full range of the galactic center $\mathrm{CO}$ emission. The antenna temperature noise level of the spectra was $\leq 0.10 \mathrm{~K}$ (rms). The data are presented in a latitude-longitude spatial map and in sets of longitudevelocity and latitude-velocity diagrams. This survey combines the characteristics of being very sensitive and well sampled, and of having one of the widest and more homogeneous spatial coverage of the region within $\sim 2 \mathrm{kpc}$ from the galactic center.
\end{abstract}

Key words: surveys - ISM: molecules - Galaxy: center - radio lines: ISM

\section{Introduction}

The central region of our Galaxy may hold the key to many questions related to the structure, kinematics, chemistry, dynamics and energetics of the whole Milky Way. Radio lines are a powerful tool to investigate this inner region since they are not affected by obscuring dust along the line of sight. Because the line profiles provide velocity information which can be used to study the kinematics of

Send offprint requests to: J. May the emitting gas, there has been a keen interest in observing the $\mathrm{HI}$ line at $21 \mathrm{~cm}$ and many molecular transitions.

Studies of the galactic central region can concentrate on the nuclear area (Sgr A complex) or on the large scale aspects of the whole central area. Although the study of the complex and unique galactic nucleus requires highresolutions observations, the large-scale properties of the central region of the Galaxy can be adequately studied in surveys made with relatively low-resolution instruments within a reasonable amount of time.

There have been several molecular line surveys of the central region of the Galaxy, for example, of the hydroxyl radical (McGee et al. 1970; Cohen \& Dent 1983; Boyce \& Cohen 1994), formaldehyde (Scoville et al. 1972; Cohen \& Few 1981), ammonia (Kaifu et al. 1975), carbon sulfide (Bally et al. 1987).

Of particular importance are the carbon monoxide surveys because this molecule is a good tracer of molecular hydrogen, which cannot be observed directly by ground based telescopes. The observations of various $\mathrm{CO}$ isotopes can yield different information on the physical state of the emitting gas. For example, observations of the optically thick CO emission can be used, in principle, to estimate the excitation temperature of a molecular cloud; however, due to the large temperature gradients that almost certainly exist, the $\mathrm{CO}$ emission can trace the excitation temperature at the $\tau=1$ surface of the cloud. Alternatively, observations of the optically thinner ${ }^{13} \mathrm{CO}$ can be used to estimate the column density. A problem with the ${ }^{13} \mathrm{CO}$ and with the $\mathrm{C}^{18} \mathrm{O}$, which is also optically thin, is that their radiation is several times weaker than that of $\mathrm{CO}$ and thus, their observations are very time consuming. However, the velocity-integrated CO intensity can be used as a measure of the column density of molecular clouds, in roughly the same way that the luminosity of a globular cluster provides a measure of its mass: by being proportional to the number column density of unresolved optically thick objects that do not seriously overlap (Bronfman et al. 1988).

Surveys of the galactic center in several CO isotopes are presented in Table 1 . This table shows that most of the 
Table 1. CO surveys of the galactic center region

\begin{tabular}{llll}
\hline Reference & Isotope & Extent in $l$ & Extent in $b$ \\
\hline Liszt \& Burton (1978) & $\mathrm{CO}(J=1 \rightarrow 0)$ & $-5.3^{\circ} \leq l \leq 10^{\circ \mathrm{a}}$ & $b=-3^{\prime},-63^{\prime}, 57^{\prime}$ \\
& & $l=-123^{\prime},-53^{\prime},-3^{\prime}, 47^{\prime}, 117^{\prime}$ & $-1.1^{\circ} \leq b \leq 1.2^{\circ \mathrm{b}}$ \\
Bania (1980) & $\mathrm{CO}(J=1 \rightarrow 0)$ & $-10^{\circ} \leq l \leq 25^{\circ}$ & $b=-20^{\prime}, 20^{\prime}$ \\
Bania (1986) & $\mathrm{CO}(J=1 \rightarrow 0)$ & $-10^{\circ} \leq l \leq 25^{\circ}$ & $b=0^{\circ},-10^{\prime}, 10^{\prime},-20^{\prime}, 20^{\prime}$ \\
Heiligman (1987) & ${ }^{13} \mathrm{CO}(J=1 \rightarrow 0)$ & $-2^{\circ} \leq l \leq 2^{\circ}$ & $-0.467^{\circ} \leq b \leq 0.467^{\circ}$ \\
Bally et al. (1987) & ${ }^{13} \mathrm{CO}(J=1 \rightarrow 0)$ & $-5^{\circ} \leq l \leq 5^{\circ}$ & $-0.6^{\circ} \leq b \leq 0.6^{\circ}$ \\
Bally J. (1996) & $\mathrm{CO}(J=1 \rightarrow 0)$ & $-2.5^{\circ} \leq l \leq 4^{\circ}$ & $-0.6^{\circ} \leq b \leq 0.6^{\circ}$ \\
Oka et al. (1996) & $\mathrm{CO}(J=2 \rightarrow 1)$ & $-3^{\circ} \leq l \leq 3^{\circ}$ & $b=0^{\circ}$ \\
Dahmen et al. $(1996)$ & $\mathrm{C} 18 \mathrm{O}(J=1 \rightarrow 0)$ & $-1.05^{\circ} \leq l \leq 3.6^{\circ}$ & $-0.9^{\circ} \leq b \leq 0.75^{\circ}$ \\
\hline
\end{tabular}

a 3 different ranges in longitude

b 5 different ranges in latitude

c 2 strips in latitude at $l=-0.5^{\circ}$ and $0.5^{\circ}$.

$\mathrm{CO}$ observations of the galactic center region have been obtained from the northern hemisphere, where the galactic center is observable only at low elevations, and with relatively high angular resolution $\left(\sim 1^{\prime}\right)$. As a consequence of these conditions, most of CO northern hemisphere observations of the molecular gas within a few kpc from the galactic center are severely under-sampled.

Well sampled observations have been restricted only to relatively small areas around the nucleus and near the galactic plane, while surveys with larger coverage consisted of longitude strips at a few fixed latitudes, with typical spacings of $0.5^{\circ}$ ( $\sim 30$ beam-widths $)$. These coarse grids can easily miss distant molecular clouds close to the galactic center. Results of the out-of-plane surveys of the inner galactic disk (Cohen et al. 1980, 1986; Grabelsky et al. 1987; Bronfman et al. 1988) have shown that well sampled observations (spaced at $\sim 1$ beamwidth) are necessary in order to follow the continuity of large scale galactic features.

The purpose of this work is to present a well sampled and sensitive large scale $\mathrm{CO}$ survey of the central region of the Galaxy, providing more complete information about the region within $2 \mathrm{kpc}$ from the center of the Galaxy. The results of this survey are well suited for direct comparison with HI surveys of our Galaxy made with the largest single-dish telescopes, and with high-resolution interferometric CO observations of other spiral galaxies. For example, the 100-m Effelsberg Telescope has a beam-width of $9^{\prime}$ (FWHM) at 21-cm wavelength.

\section{Instrumentation}

The survey was carried out with the Southern Millimeterwave Telescope (Cohen 1983; Bronfman et al. 1988, 1989) located at Cerro Tololo Interamerican Observatory, Chile. The telescope is a 1.2-m Cassegrain with a beamwidth of $8.8^{\prime}$ (FWHM) at $115 \mathrm{GHz}$, the frequency of the
$\mathrm{CO}(J=1 \rightarrow 0)$ transition. It was equipped with a super-heterodyne receiver with a SSB noise temperature of $380 \mathrm{~K}$. The first stage of the receiver consisted of a Schottky barrier diode mixer and a GaAs field-effect transistor amplifier cooled to $77 \mathrm{~K}$ by liquid nitrogen.

The spectrometer was a 256-channel filter bank of standard NRAO design. Each filter, $0.5 \mathrm{MHz}$ wide, provided a velocity resolution of $1.3 \mathrm{~km} \mathrm{~s}^{-1}$ at $115 \mathrm{GHz}$ and a coverage of $333 \mathrm{~km} \mathrm{~s}^{-1}$. This is adequate for most of the galactic $\mathrm{CO}$ emission, except within the central few degrees $\left(-4.5^{\circ}<l \leq+5^{\circ}\right)$ where the velocity range of the emission reaches $500 \mathrm{~km} \mathrm{~s}^{-1}$. In such region two spectra centered at different VLSRs were taken at each position and were subsequently combined (Table 2).

Table 2. Parameters of the survey

\begin{tabular}{ll}
\hline Galactic longitude & $-12^{\circ}$ to $+13^{\circ}$ \\
Galactic latitude & $-2^{\circ}$ to $+2^{\circ}$ \\
Sampling interval & $7.5^{\prime}(0.85$ beam-widths $)$ for $|b| \leq 1^{\circ}$ \\
& $15^{\prime}(1.7$ beam-widths $)$ for $1^{\circ}<|b| \leq 2^{\circ}$ \\
Telescope beam & $8.8^{\prime}$ at $115 \mathrm{GHz}$ \\
(FWHM) & \\
Velocity range & $-244,+88 \mathrm{~km} \mathrm{~s}^{-1}\left(-12^{\circ} \leq l \leq-6.5^{\circ}\right)$ \\
& $-179,+153 \mathrm{~km} \mathrm{~s}^{-1}\left(-6.5^{\circ}<l \leq-4.5^{\circ}\right)$ \\
& $-319,+319 \mathrm{~km} \mathrm{~s}^{-1}\left(-4.5^{\circ}<l \leq+5^{\circ}\right)$ \\
& $-88,+244 \mathrm{~km} \mathrm{~s}^{-1}\left(+5^{\circ}<l \leq+13^{\circ}\right)$ \\
Velocity resolution & $1.3 \mathrm{~km} \mathrm{~s}^{-1}$ \\
RMS sensitivity & $\leq 0.10 \mathrm{~K}\left(1 \sigma\right.$ in $T_{\mathrm{A}}^{*}$ at $\left.1.3 \mathrm{~km} \mathrm{~s}^{-1}\right)$ \\
\hline
\end{tabular}

The system was calibrated daily and checked against reference sources before starting the observations. A standard blackbody chopper-wheel technique (Kutner \& Ulich 1981) was used to produce antenna temperatures, $T_{\mathrm{A}}^{*}$, corrected for atmospheric attenuation, resistive losses, and rearward spillover and scattering. To correct for forward 
spillover and scattering, $T_{\mathrm{A}}^{*}$ is divided by the main beam efficiency, $\eta$, the fraction of the forward power that enters the main beam. This yields the radiation temperature $T_{\mathrm{R}}=T_{\mathrm{A}}^{*} / \eta$, the physical temperature of a blackbody that just fills the main beam. The main beam efficiency of the telescope, $\eta=0.82$, has been derived by Bronfman et al. (1988).

The atmospheric conditions at Cerro Tololo are extremely favorable for mm-wave observations, the water vapor opacity per air mass being usually less than 0.1. Since the galactic center transits there almost at the zenith, most of the spectra were taken through an air mass of about 1 . These circumstances made possible, even with a Schottky receiver, to reach rms antenna noise temperatures of $\leq 0.10 \mathrm{~K}$ in 10 minutes or less.

The pointing of the telescope was checked twice a day by observing the Sun. Right ascension and declination scans across the Sun's limb were used to locate the center of the solar disk in $115 \mathrm{GHz}$ continuum emission to within $15^{\prime \prime}$. The long term pointing accuracy was checked every few months by observing stars with a small optical telescope collimated with the radio telescope; errors smaller than $1^{\prime}$ were obtained.

Computer control of the dome and of the altitudeazimuth mount of the telescope allowed fast pointing changes which made practical to have reference positions several degrees away from the galactic equator.

Table 3. Emission-free reference positions

\begin{tabular}{llllll}
\hline$l$ & $b$ & $l$ & $b$ & $l$ & $b$ \\
$\left(^{\circ}\right)$ & $\left(^{\circ}\right)$ & $\left(^{\circ}\right)$ & $\left(^{\circ}\right)$ & $\left(^{\circ}\right)$ & $\left(^{\circ}\right)$ \\
\hline 347.00 & +3.00 & 357.00 & -5.00 & 5.00 & -4.00 \\
348.00 & -5.00 & 357.00 & +3.00 & 7.00 & +5.00 \\
348.00 & -4.00 & 359.80 & -5.75 & 8.00 & -5.00 \\
352.00 & -5.00 & 0.00 & -4.00 & 8.50 & +2.75 \\
353.00 & -5.00 & 0.00 & -3.00 & 9.00 & +5.00 \\
353.00 & +4.00 & 0.70 & -3.30 & 10.00 & +5.00 \\
354.00 & -5.00 & 1.00 & -3.90 & 11.00 & -4.00 \\
355.00 & -5.00 & 1.90 & -5.35 & 12.00 & +5.00 \\
355.00 & +5.00 & 3.80 & -4.80 & 14.00 & +2.50 \\
356.04 & -5.23 & 4.75 & +2.50 & 15.00 & -5.00 \\
\hline
\end{tabular}

\section{Observations}

The survey consists of almost 8000 spectra which were taken between February and November 1984. The area observed covered $-12^{\circ} \leq l \leq+13^{\circ}$ and $-2^{\circ} \leq b \leq+2^{\circ}$. Spectra were obtained, both in longitude and latitude, every $7.5^{\prime}$ ( 0.85 beam-widths) for $|b| \leq 1^{\circ}$, and every $15^{\prime}(1.7$ beam-widths) for $1^{\circ}<|b| \leq 2^{\circ}$.

Each spectrum was integrated for about 10 minutes, to yield an antenna temperature noise level of $\leq 0.10 \mathrm{~K}(\mathrm{rms})$ for a $1.3 \mathrm{~km} \mathrm{~s}^{-1}$ velocity resolution (Table 2). In order
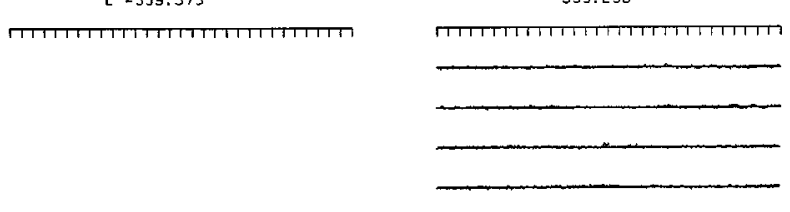

1.250

1.000

0.875

0.750

0.625

0.375

0.250

0.125

0.0

$-0.125$

$-0.250$

$-0.375$

$-0.500$

$-0.625$

$-0.750$

$-0.875$

$-1.000$

$-1.250$

$-1.500$

$-1.750$

$-2.000$
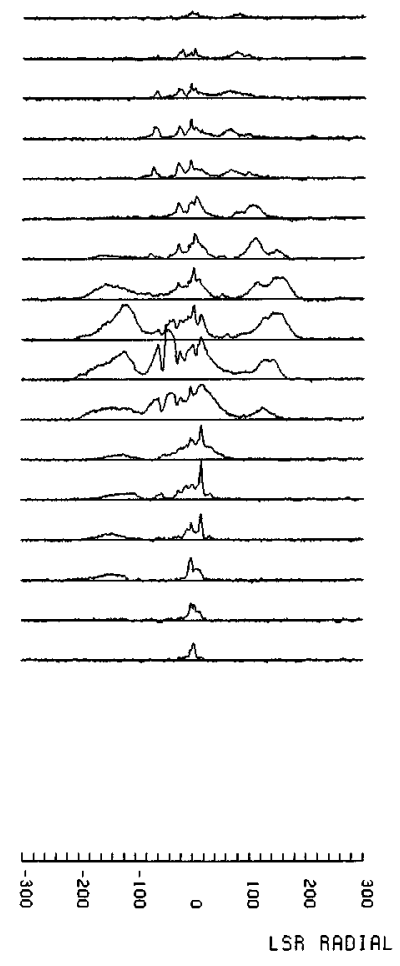

Fig. 1. Representative survey spectra along two adjacent longitude strips, illustrating their high signal-to-noise ratios and flat baselines. The baselines are spaced by $6 \mathrm{~K}$. Notice that even in this case where two overlapping spectra, $333 \mathrm{~km} \mathrm{~s}^{-1}$ wide each, were combined to achieve the necessary velocity range, the baselines are still perfectly flat. Also the difference in sampling for $|b|>1^{\circ}$ can be clearly seen

to subtract instrumental effects and the sky background the antenna was switched, every 15 seconds, between the target position (on) and an emission-free position (off) at elevations closer than $1^{\circ}$. The off positions were verified to have emission not greater than $0.04 \mathrm{~K}$ (Bitran 1987). Table 3 lists the emission-free positions.

Observations were carried out only under good weather conditions, i.e. when the zenith opacity of water vapor at $115 \mathrm{GHz}$ was between 0.05 and 0.20 . During the observations the spectra were examined visually and fitted with linear baselines only. If a spectrum showed evidence of baseline distortion it was discarded and the observation repeated.

Within $5^{\circ}$ of the galactic center the velocity span of the $\mathrm{CO}$ emission exceeded the full range of the spectrometer. This problem was circumvented by taking, for all positions within $-4.5^{\circ}<l \leq+5^{\circ}$, two spectra centered at velocities $306 \mathrm{~km} \mathrm{~s}^{-1}$ apart (Table 2). These two spectra 

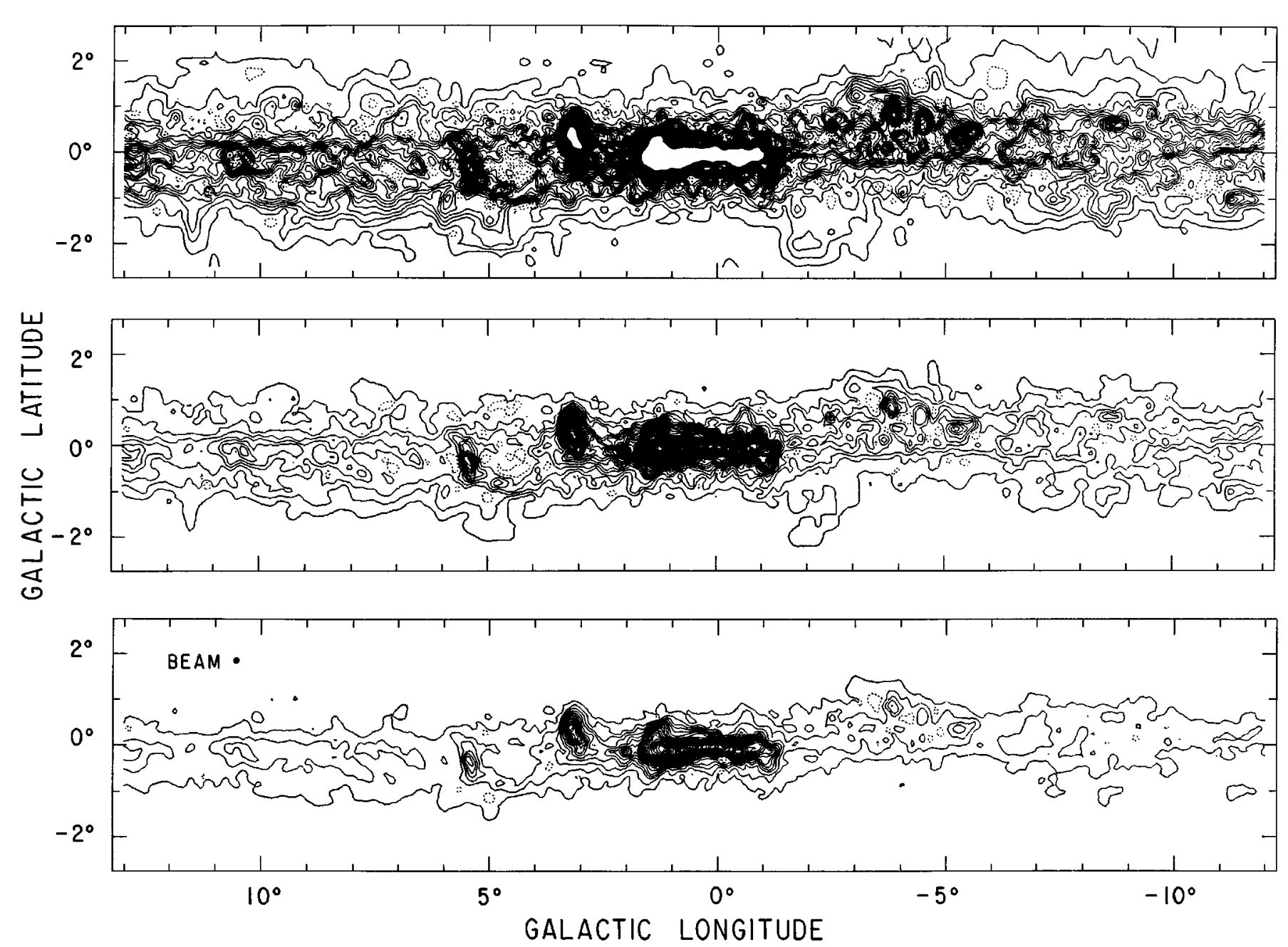

Fig. 2. Spatial maps of CO line intensity $\int T_{\mathrm{A}}^{*} \mathrm{~d} v$ integrated over the velocity range of -320 to $320 \mathrm{~km} \mathrm{~s}^{-1}$ displayed at three different contour levels. The contour intervals and the lowest contours have been set at $13,32.5$ and $65 \mathrm{~K} \mathrm{~km} \mathrm{~s}^{-1}$, corresponding to $\sim 4 \sigma, \sim 10 \sigma$, and $\sim 20 \sigma$, in the top, middle and bottom maps, respectively

covered a combined range of $640 \mathrm{~km} \mathrm{~s}^{-1}$ with an overlap of $26 \mathrm{~km} \mathrm{~s}^{-1}$ (20 channels) allowing ample emission-free sections to set baselines, and enough overlap to check that the spectra matched properly (Fig. 1). The emission level in the overlapping channels was checked to coincide in both spectra within the antenna temperature rms noise. Poor matchings were rejected and the corresponding positions re-observed. A sample of combined spectra along two adjacent longitude strips are shown in Fig. 1; the high signal-to-noise ratio and the flat baseline of these spectra are characteristics of the whole survey.

An important goal of this survey was to cover the full latitude extent of the galactic $\mathrm{CO}$ emission. We found most of the emission to be confined in $|b| \leq 1^{\circ}$, therefore our latitude coverage $\left(|b| \leq 2^{\circ}\right)$ seems adequate. However, because $\mathrm{HI}$ emission is observed at higher latitudes in opposite galactic quadrants (at $b>0^{\circ}$ for $l<0^{\circ}$, and at $b<0^{\circ}$ for $l>0^{\circ}$ ), we conducted a search between $b=2^{\circ}$ and $b=2.5^{\circ}$ for $l<0^{\circ}$, and between $b=-2^{\circ}$ and $b=-2.5^{\circ}$ for $l>0^{\circ}$, detecting only local emission.

\section{Data presentation}

The basic data of the survey are the spectra obtained point by point in longitude and latitude, comprising therefore a data cube or three dimensional array of antenna temperatures. A two dimensional representation of the array was obtained by integrating the emission over the whole velocity span, between -320 and $+320 \mathrm{~km} \mathrm{~s}^{-1}$, to produce a spatial map useful to compare the molecular gas distribution, as traced by the $\mathrm{CO}(J=1 \rightarrow 0)$ transition, with that of infrared and radio continuum emission. Such map is shown in Fig. 2, where the contour interval is set to $65 \mathrm{~K} \mathrm{~km} \mathrm{~s}^{-1}$.

The data are further displayed in longitude-velocity diagrams of the $\mathrm{CO}$ emission (Fig. 3), one for each 


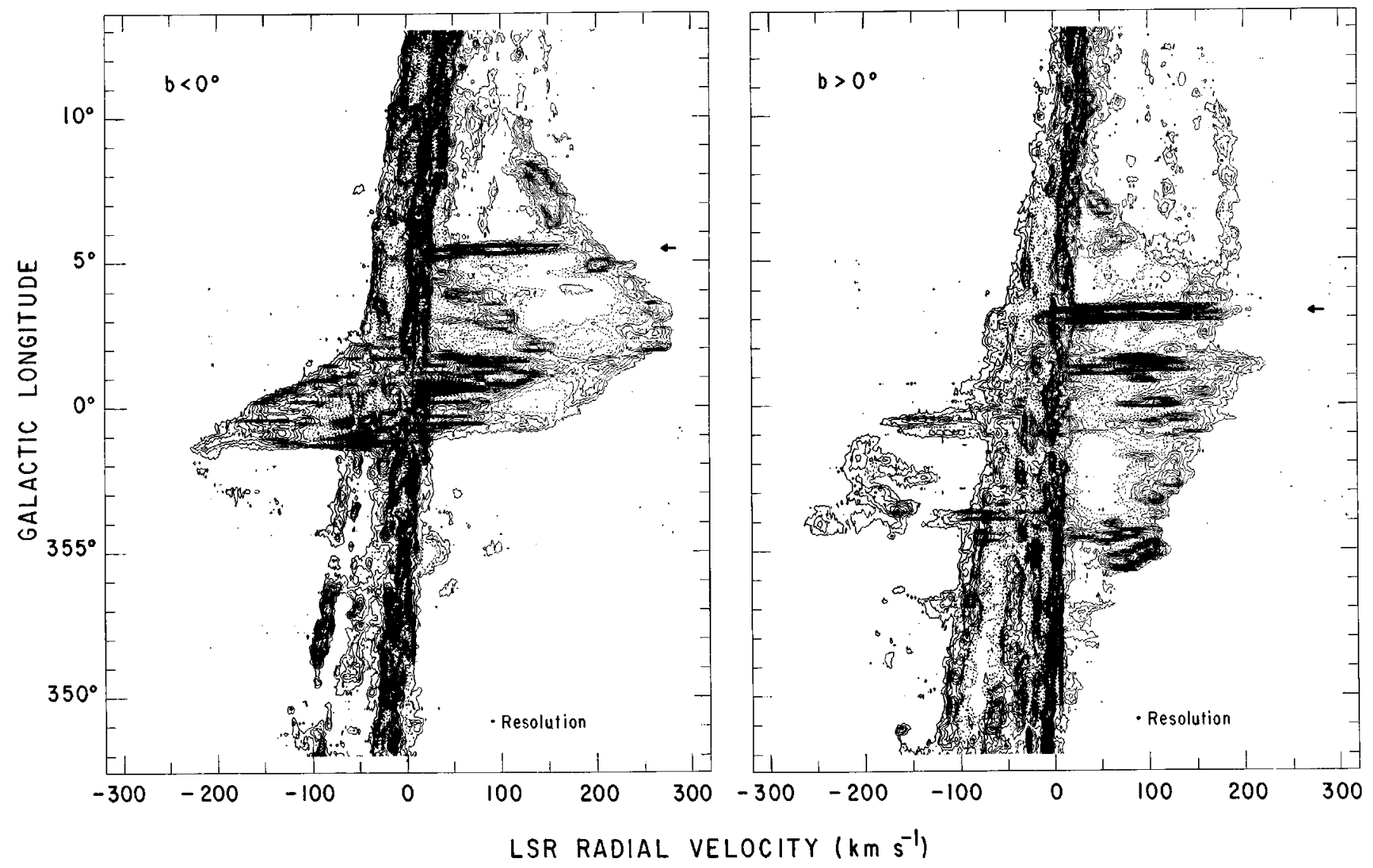

Fig. 5. Two longitude-velocity diagrams integrated below and above the galactic plane. The contour interval is $2 \mathrm{~K}$, with the lowest contour at $2 \mathrm{~K}$. The arrows point to two intense sources with unusually large velocity widths

observed latitude. This representation is particularly useful to identify kinematic structures and follow their development with galactic latitude. Contours are separated by $1 \mathrm{~K}$, and the lowest contour is set at $0.5 \mathrm{~K}$. The latitude structure of the $\mathrm{CO}$ emission is presented in latitudevelocity diagrams (Fig. 4), one for each observed longitude, with the same contour levels of Fig. 3. The velocity and latitude limits of the observations are indicated by a broken line.

\section{Discussion}

Most of the $\mathrm{CO}$ emission in this survey is contained in a band which extends trough all observed longitudes (Fig. 2). The emission occurs preferentially above the plane for negative longitudes and below the plane for positive longitudes. This asymmetry, also observed in HI (Liszt \& Burton 1980), results from a tilt in the gas distribution with respect to the galactic plane.

The CO emission is distributed closely along the galactic plane with the highest intensity concentrated between $l=-1.5^{\circ}$ and $l=1.8^{\circ}$ and within approximately $0.6^{\circ}$ of the galactic plane. This central source encompasses the large molecular complexes coincident with the major continuum sources Sgr A, B2, C and D, the rotating nuclear disk, and the expanding molecular ring, which are more clearly demarcated in the longitude-velocity diagrams (Bitran 1987). The strongly peaked central source appears very asymmetrical in galactic longitude, with most of the emission found at positive longitudes, resulting in an intensity-weighted mean longitude of $0.4^{\circ}$. The central source is also asymmetric in galactic latitude, with most of the $\mathrm{CO}$ emission originating below the IAU galactic plane, yielding an intensity-weighted mean latitude of $-0.05^{\circ}$.

The most intense $\mathrm{CO}$ emission outside the central source originates from two features with emission peaks at $l=3.15^{\circ}, b=0.3^{\circ}$ (Clump 2) and at $l=5.3^{\circ}, b=-0.3^{\circ}$ (Fig. 5). These sources are remarkable not only for their high intensities, but also for their unusually large velocity width.

Owing to its sampling, sensitivity, and extended latitude coverage, the present survey reveals new $\mathrm{CO}$ features and shows the molecular counterparts of several classic $\mathrm{HI}$ features in the inner $4 \mathrm{kpc}$ of the Galaxy. A complete 
analysis of these data, as well as a comparison with other isotopic species, will be made elsewhere.

Acknowledgements. We thank M. Koprucu for helping with some of the observations, and F. Avilés and J. Montani for the telescope maintenance. We are specially grateful to the referee, Dr. J. Bally, for his useful comments. The hospitality of Cerro Tololo Interamerican Observatory is highly appreciated. J.M., H.A. and L.B. acknowledge partial support from FONDECYT-Chile through grant 1950570.

\section{References}

Bally J., Stark A.A., Wilson R.W., Henkel C., 1987, ApJS 65, 13

Bally J., 1996, in: Gredel R. (ed.) The Galactic Center, 4th ESO/CTIO Workshop, PASPC 102. ASP, San Francisco, p. 8

Bania T.M., 1980, ApJ 242, 95

Bania T.M., 1986, ApJ 308, 868

Bitran M., 1987, Ph.D. thesis, University of Florida

Boyce P.J., Cohen R.J., 1994, A\&AS 107, 563

Bronfman L., Cohen R.S, Alvarez H., May J., Thaddeus P., 1988, ApJ 324, 248

Bronfman L., Alvarez H., Cohen R.S., Thaddeus P., 1989, ApJS 71, 481
Cohen R.J., Few R.W., 1981, MNRAS 194, 711

Cohen R.J., Dent W.R.F., 1983, in: Burton W.B., Israel F.P. (eds.) Surveys of the Southern Galaxy. Reidel, Dordrecht, p. 159

Cohen R.S., Cong H., Dame T.M., Thaddeus P., 1980, ApJ 239, L53

Cohen R.S., 1983, in: Burton W.B., Israel F.P. (eds.) Surveys of the Southern Galaxy. Reidel, Dordrecht, p. 265

Cohen R.S., Dame T.M., Thaddeus P., 1986, ApJS 60, 695

Dahmen G., Hüttemeister S., Wilson T.L., et al., 1997, A\&A (in press)

Grabelsky D.A., Cohen R.S., Bronfman L., Thaddeus P., May J., 1987, ApJ 315, 122

Heiligman G.M., 1987, ApJ 314, 747

Kaifu N., Morris M., Palmer P., Zuckerman B., 1975, ApJ 201, 98

Kutner M.L., Ulich B.L., 1981, ApJ 250, 341

Liszt H.S., Burton W.B., 1978, ApJ 226, 790

Liszt H.S., Burton W.B., 1980, ApJ 236, 779

McGee R.X., Brooks J.W., Sinclair M.W., Batchelor R.A., 1970, Aust. J. Phys. 23, 777

Oka T., Hasegawa T., Handa T., Hayashi M., Sakamoto S., 1996, ApJ 460, 334

Scoville N.Z, Solomon P.M., Thaddeus P., 1972, ApJ 172, 335 

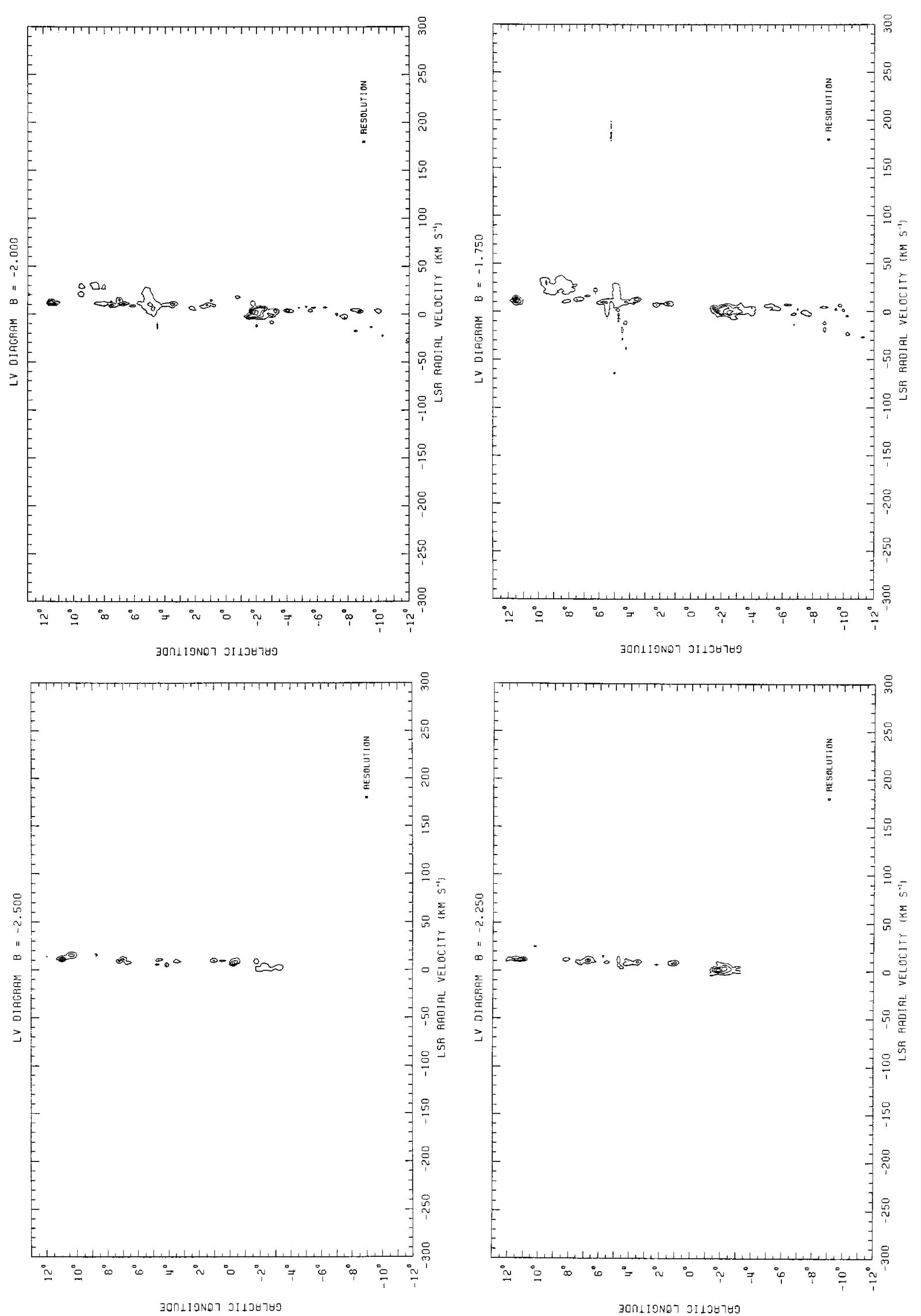

Fig. 3. Complete sequence of longitude-velocity contour diagrams, one for each latitude observed. The contoured quantity is $T_{\mathrm{A}}^{*}$. The contour interval is $1 \mathrm{~K}(10 \sigma)$, with the lowest contour at $0.5 \mathrm{~K}(5 \sigma)$. Dotted contours indicate enclosed depressions 

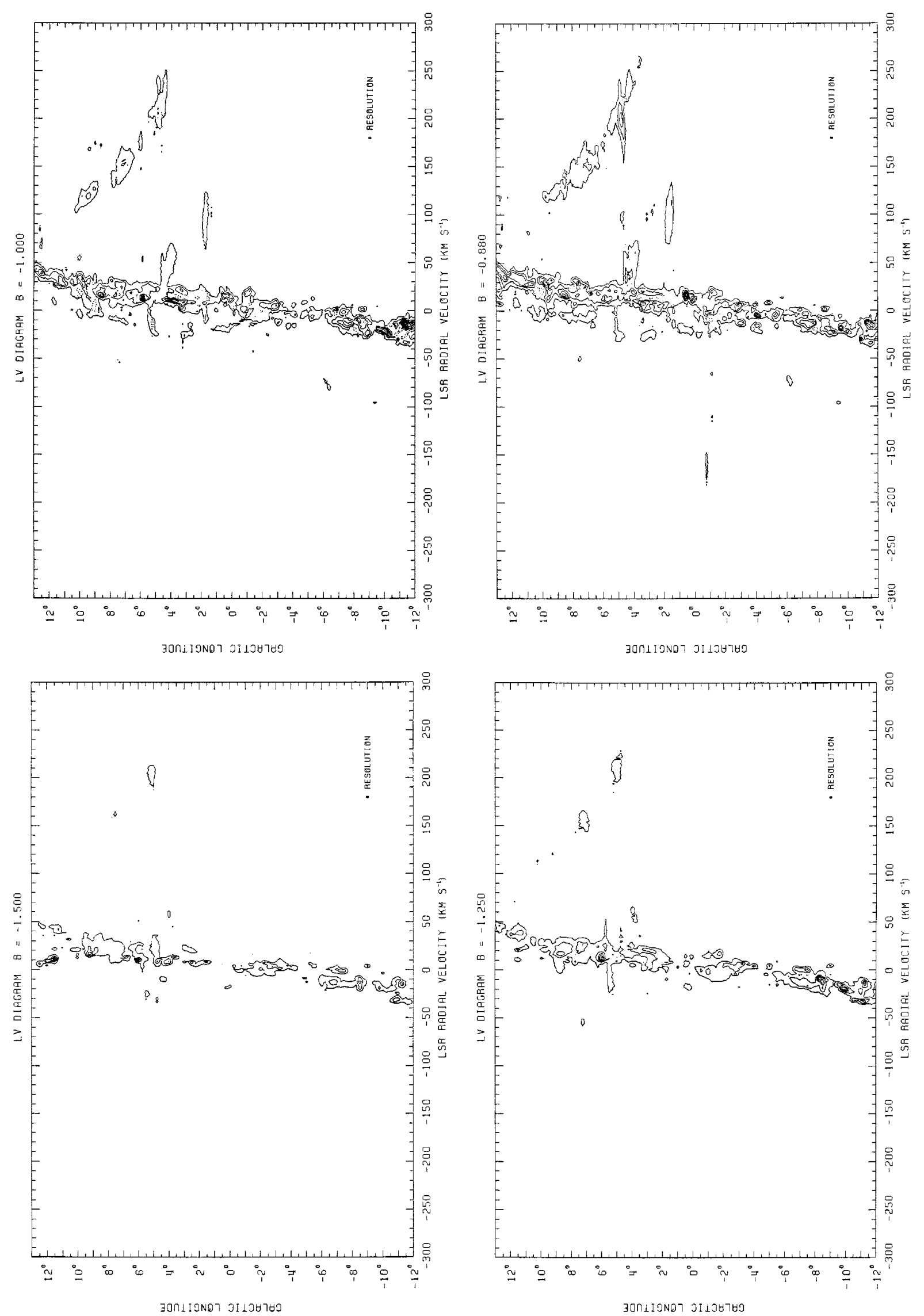

Fig. 3. continued 

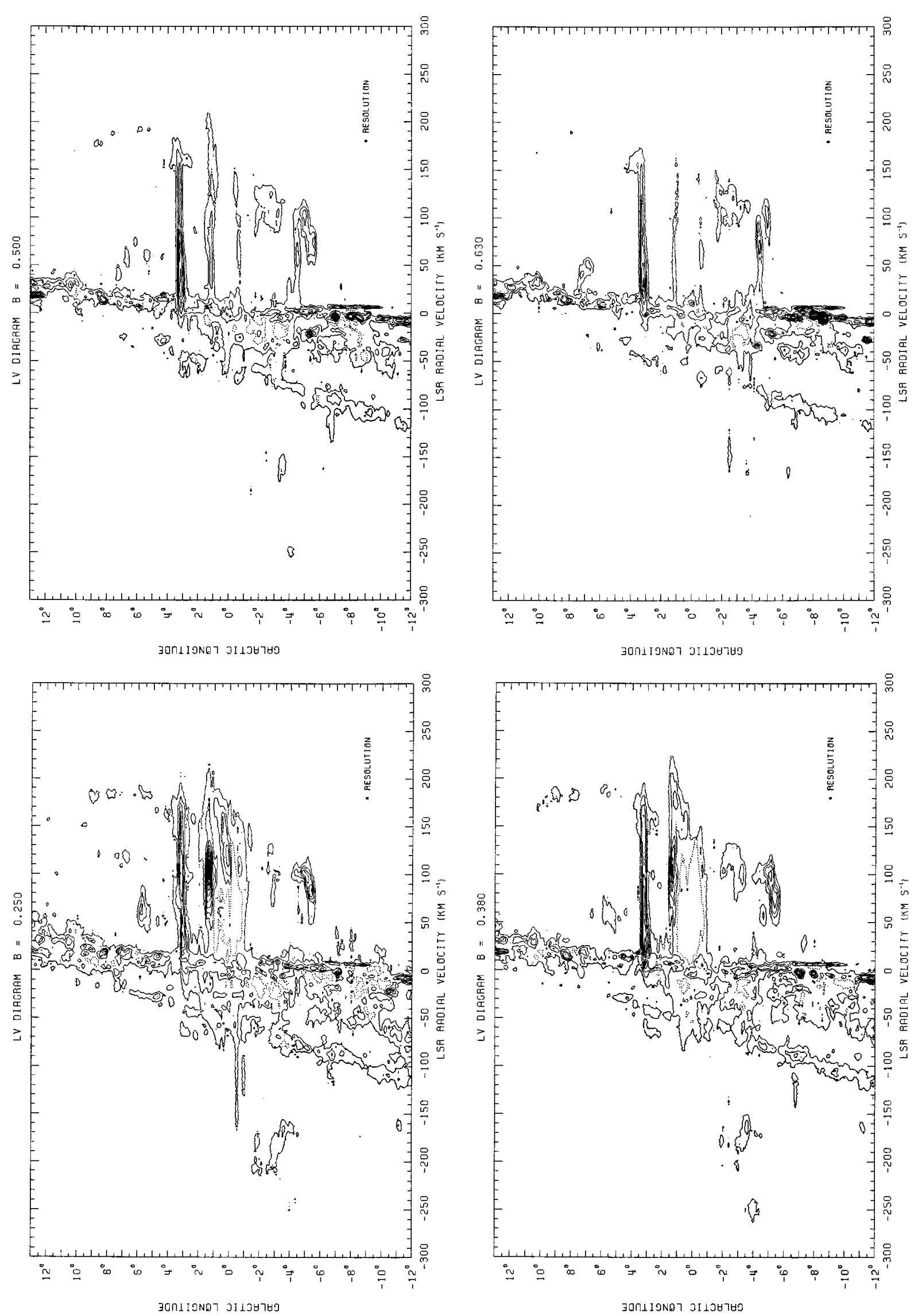

Fig. 3. continued 

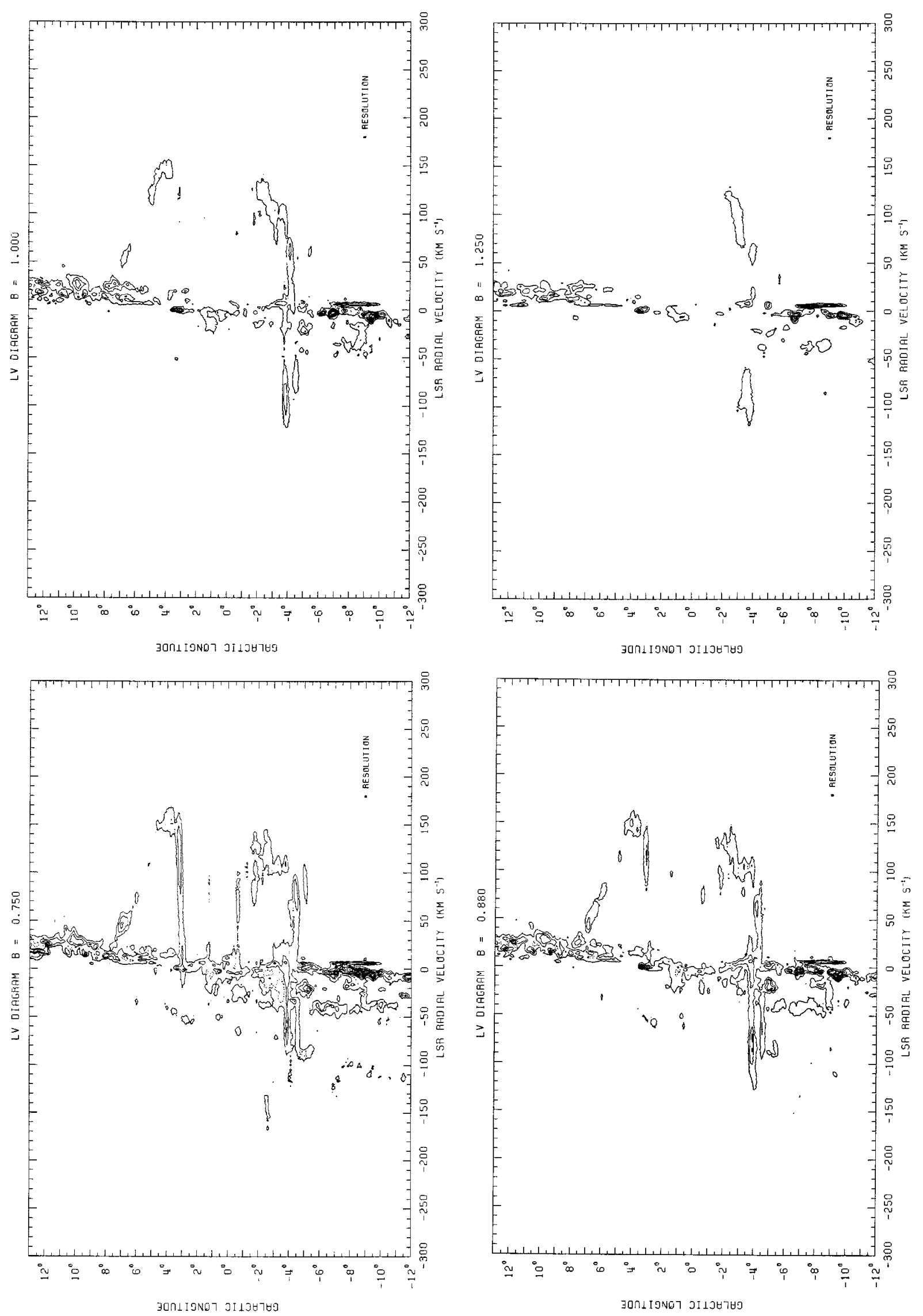

Fig. 3. continued 


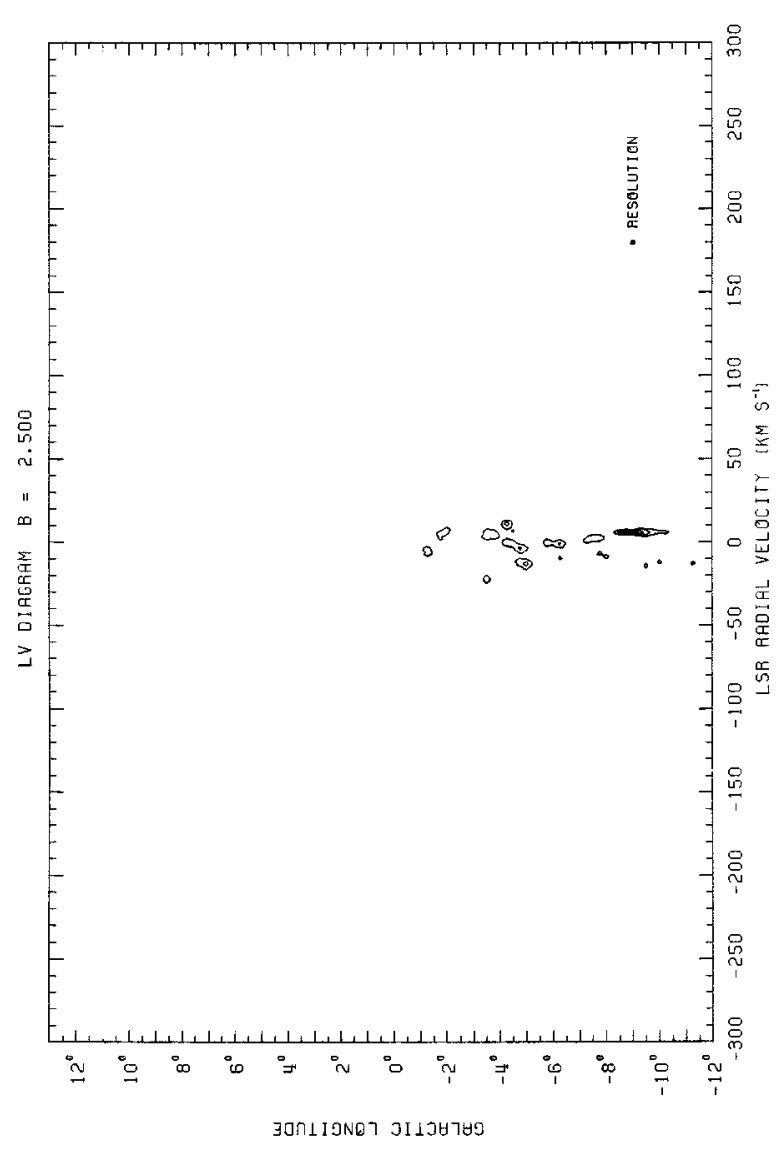

Fig. 3. continued 

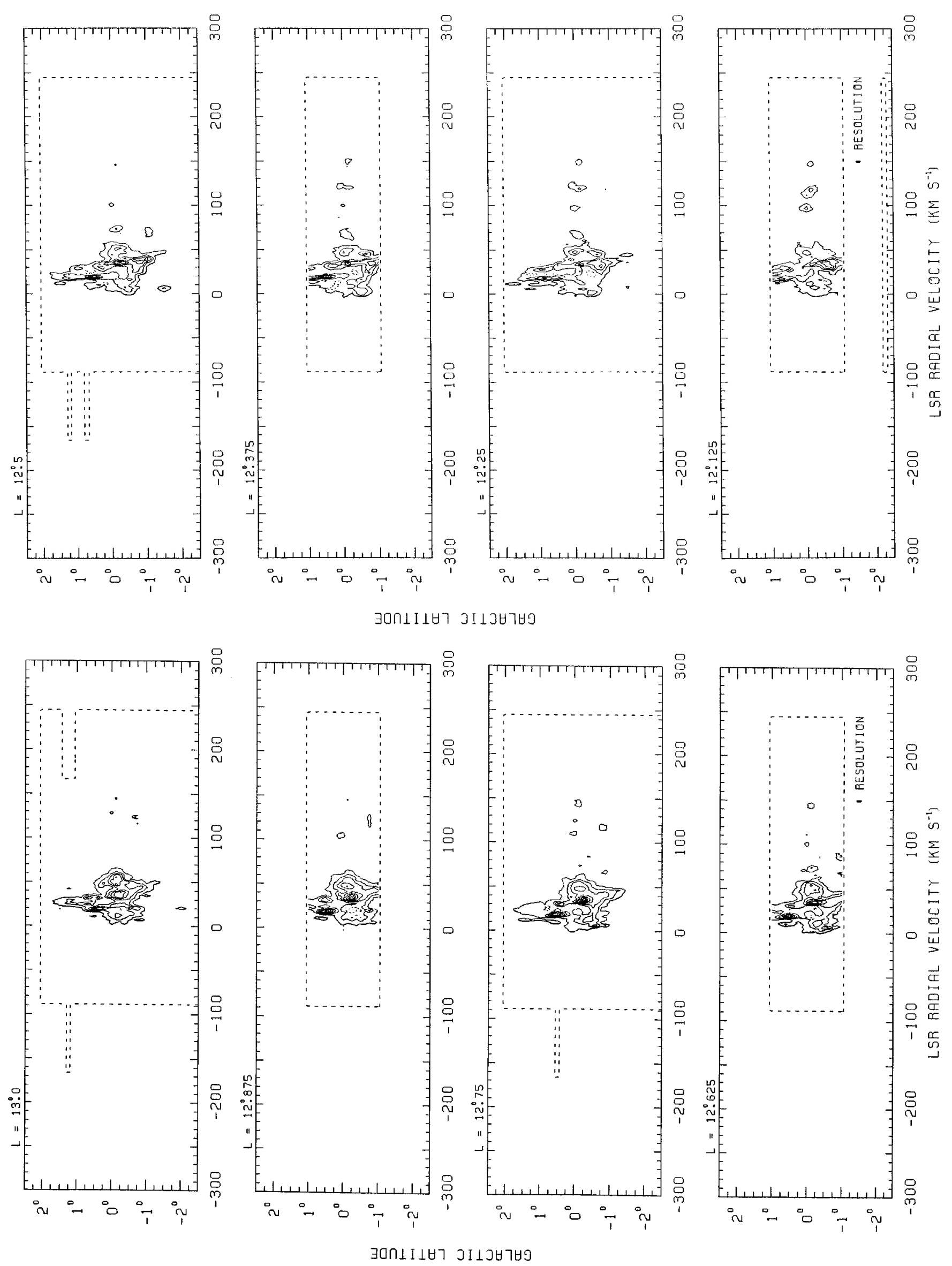

Fig. 4. Complete series of latitude-velocity contour diagrams, one for each longitude observed. The contoured quantity is $T_{\mathrm{A}}^{*}$. The contour interval is $1 \mathrm{~K}(10 \sigma)$, with the lowest contour at $0.5 \mathrm{~K}(5 \sigma)$. Dotted contours indicate enclosed depressions. The velocity and latitude coverage of the survey are indicated by a broken line 

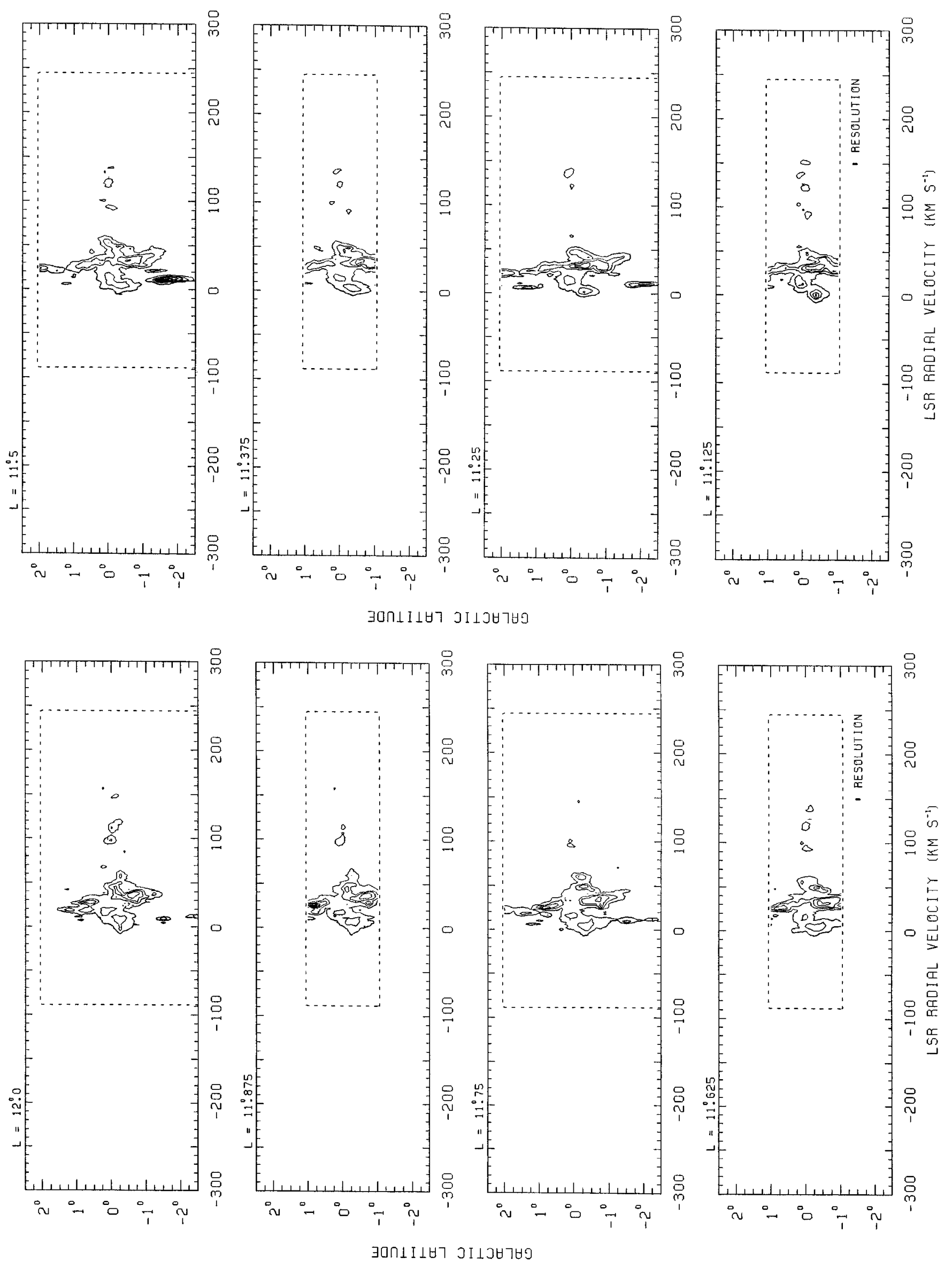

Fig. 4. continued 

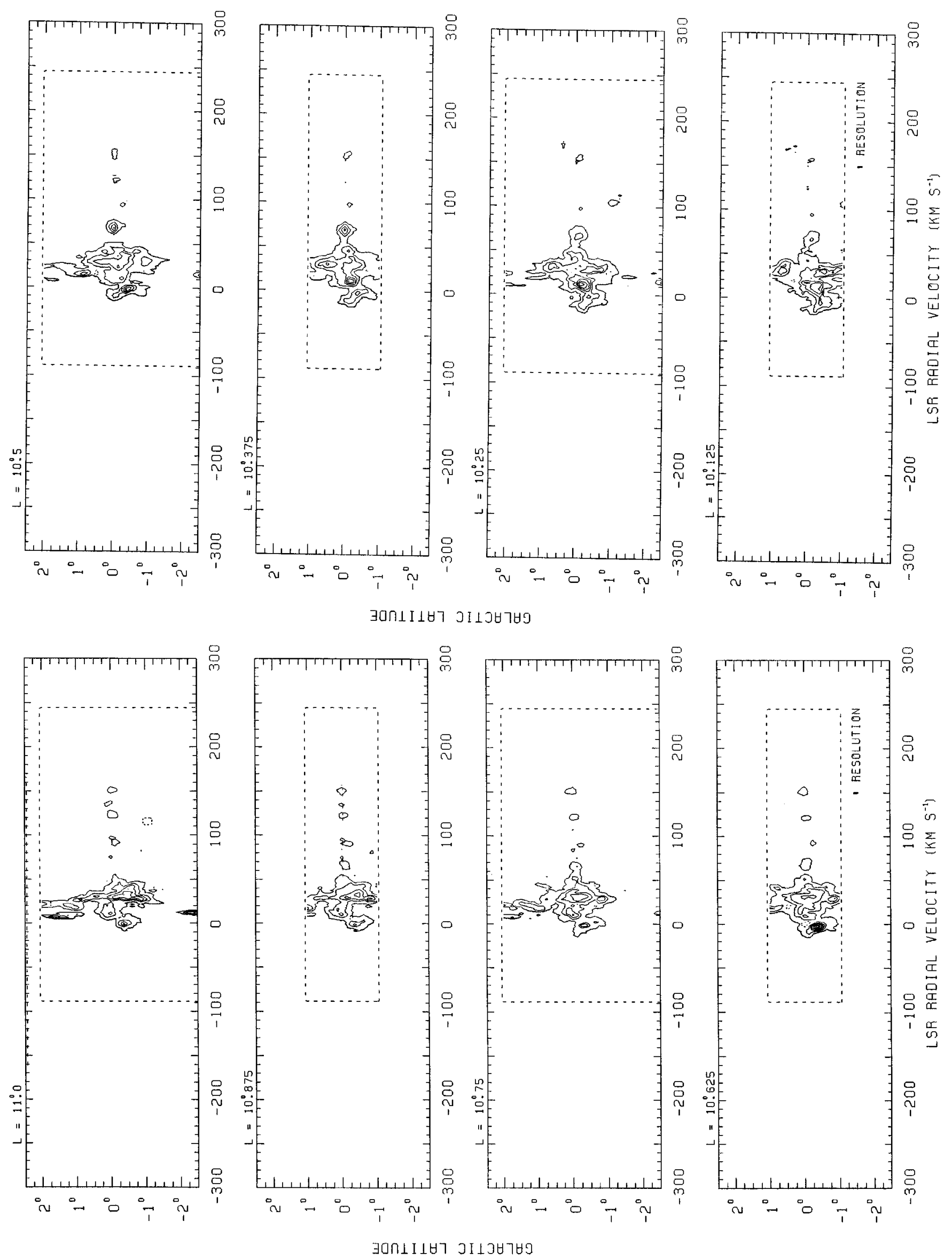

Fig. 4. continued 

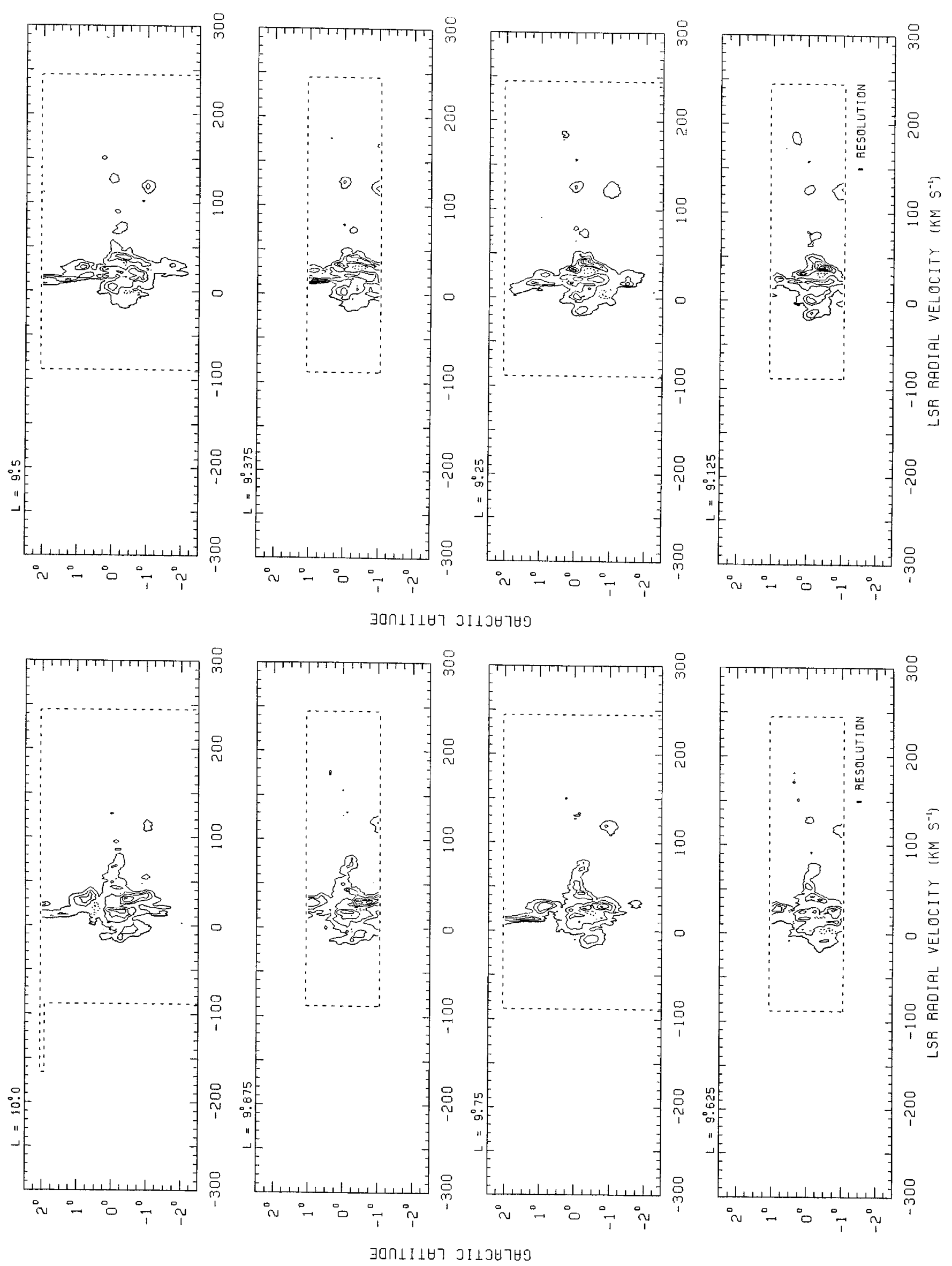

Fig. 4. continued 


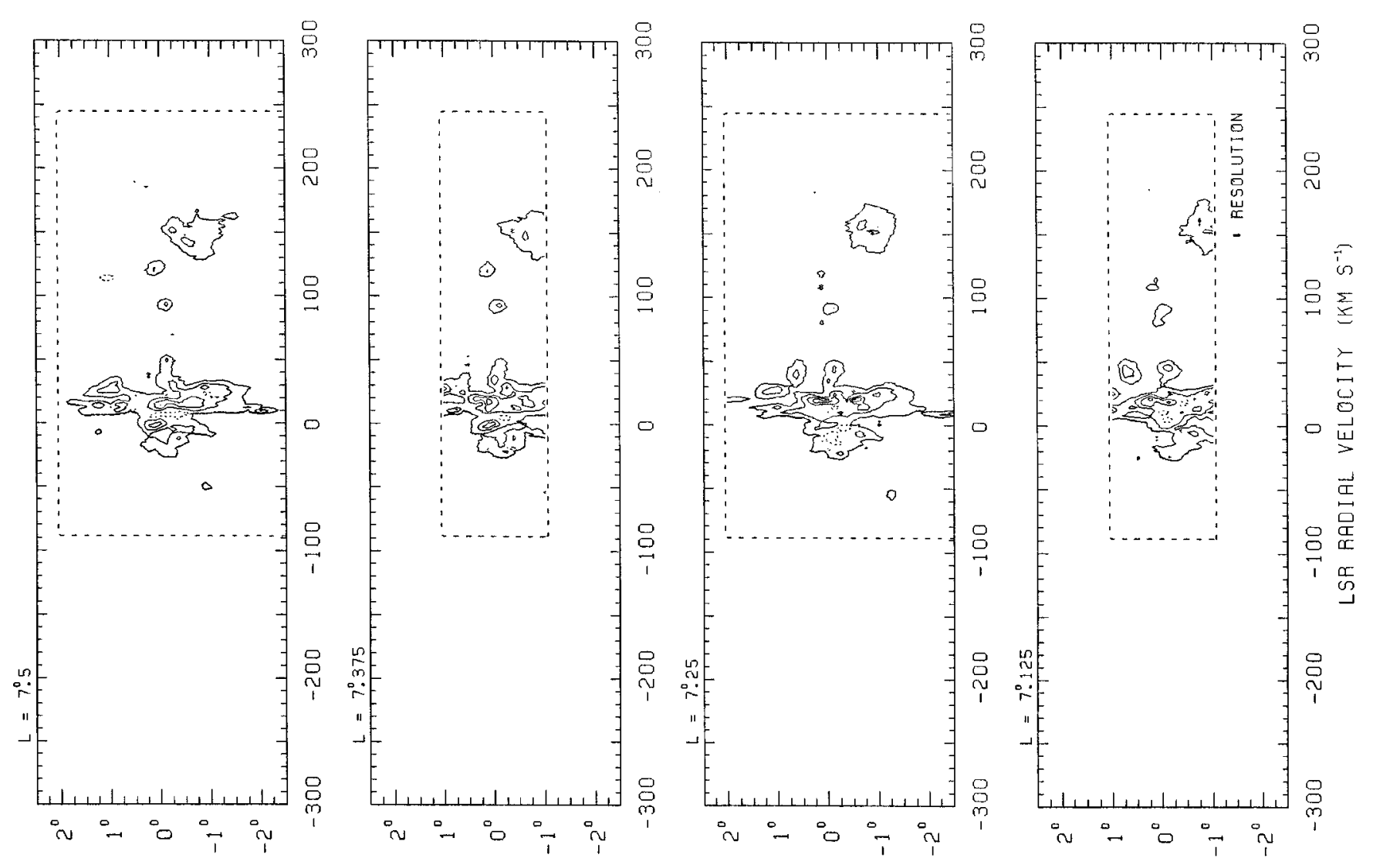

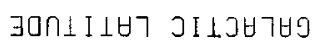
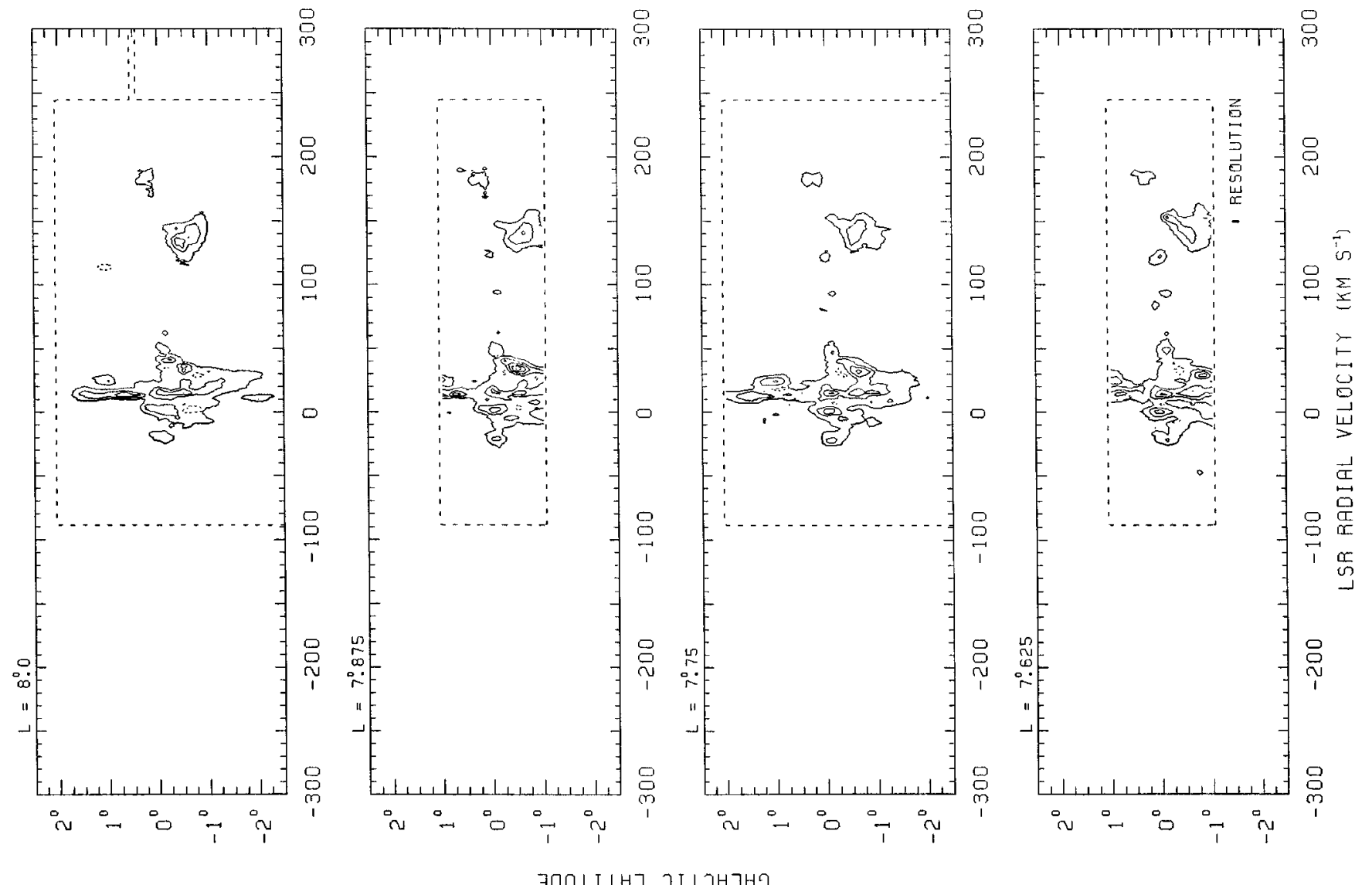

Fig. 4. continued 


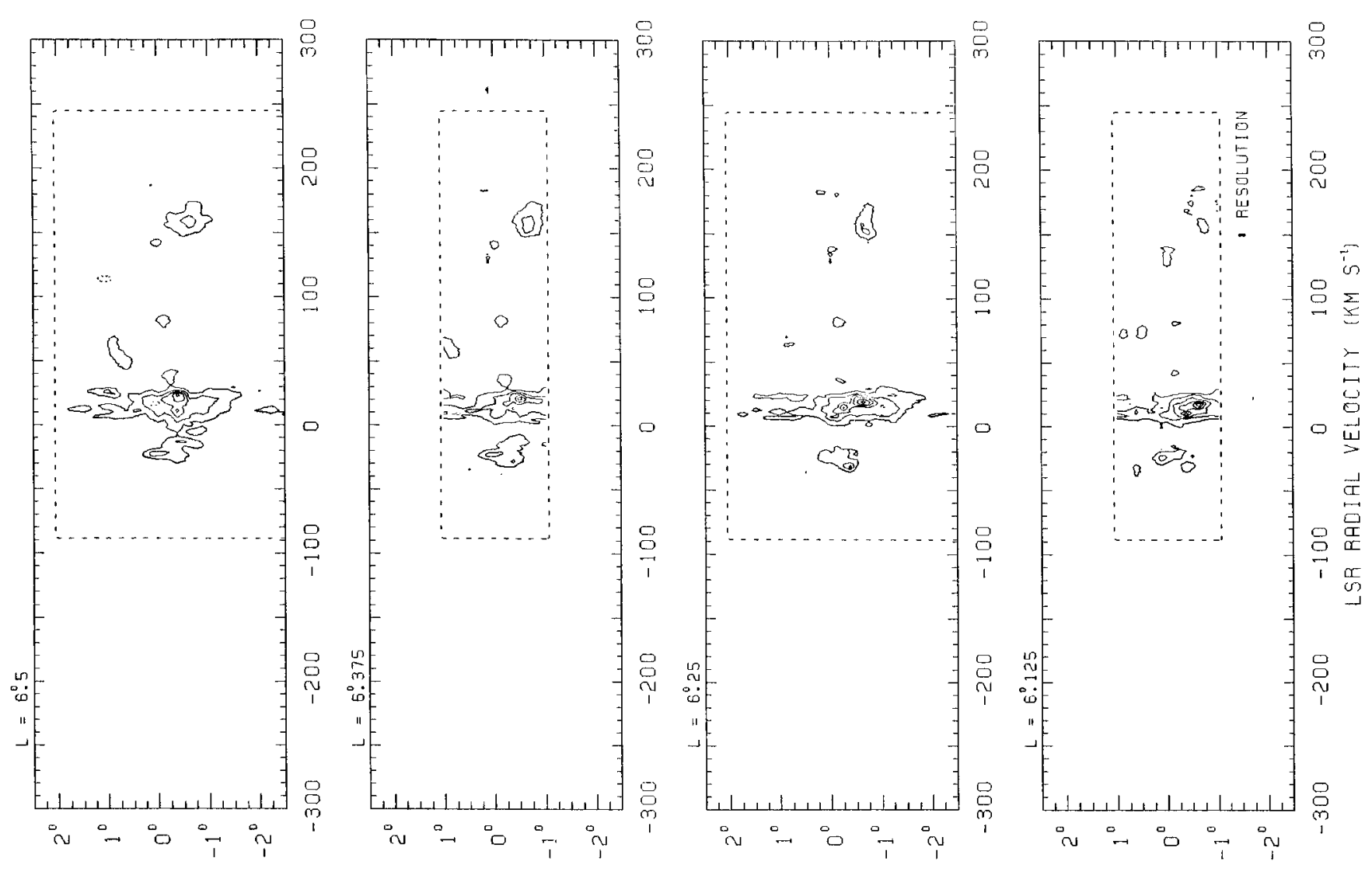

$\exists \square ก \perp \perp \forall\urcorner$ งIเวHาษฮ

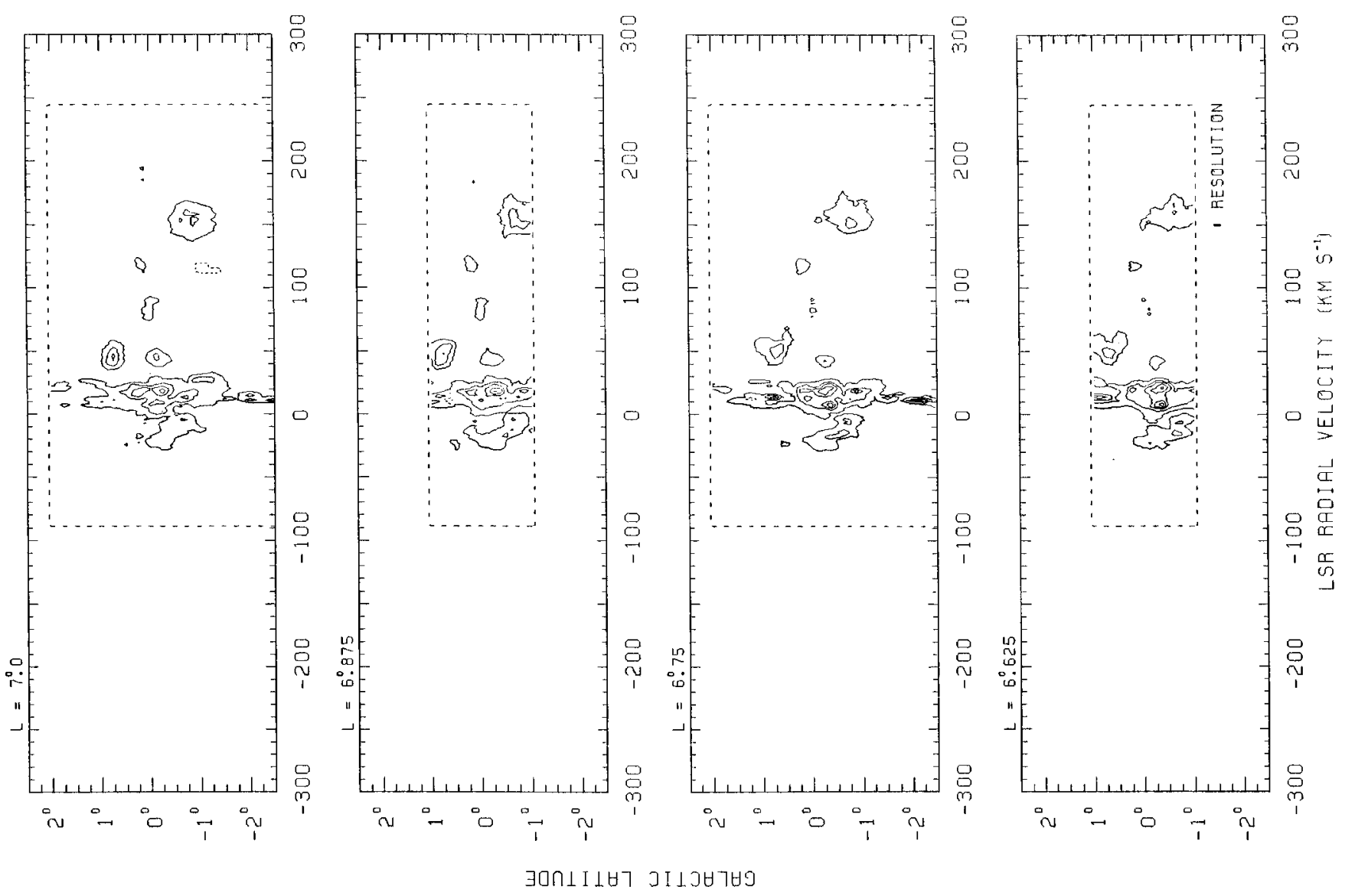

Fig. 4. continued 

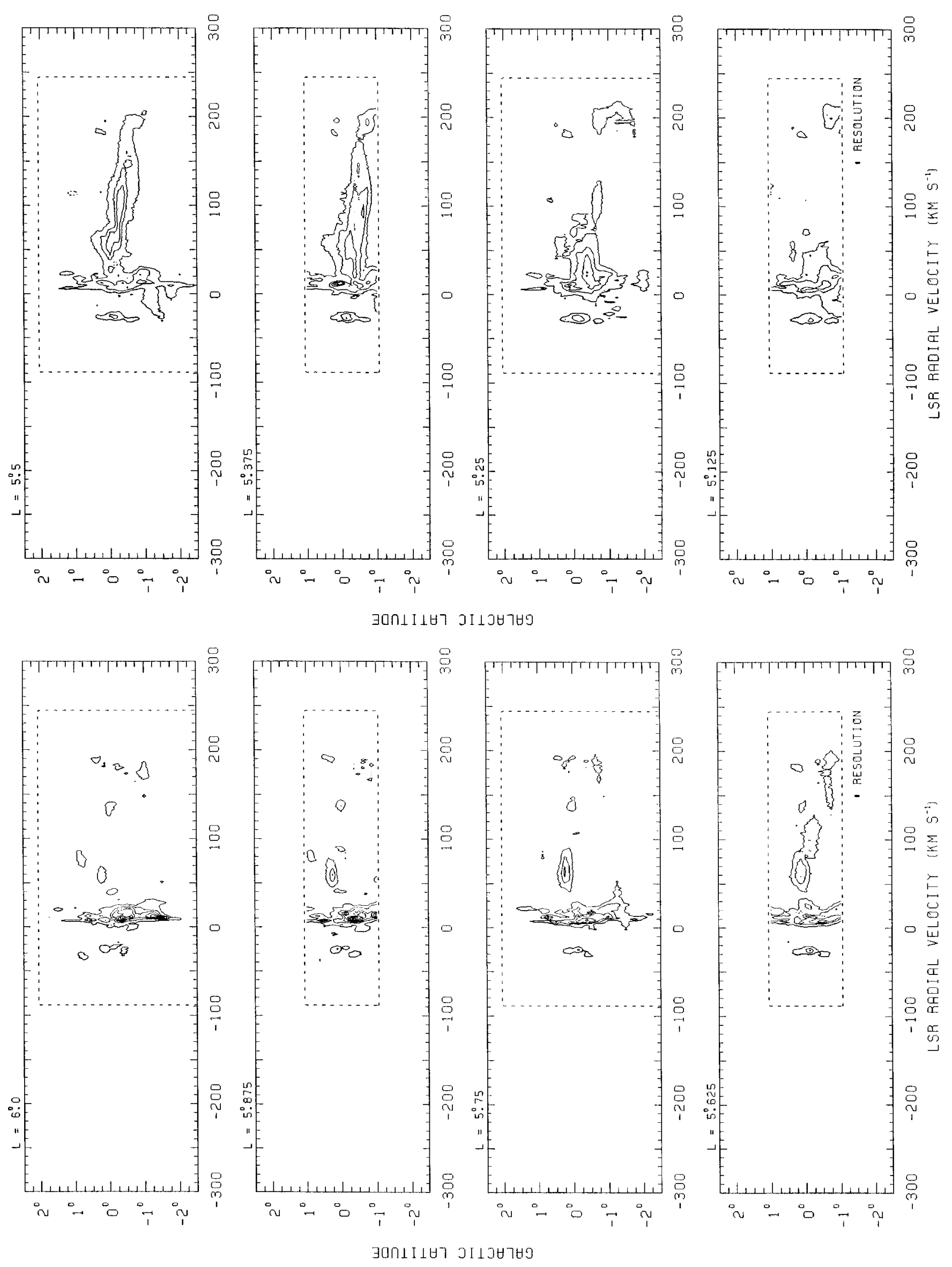

Fig. 4. continued 

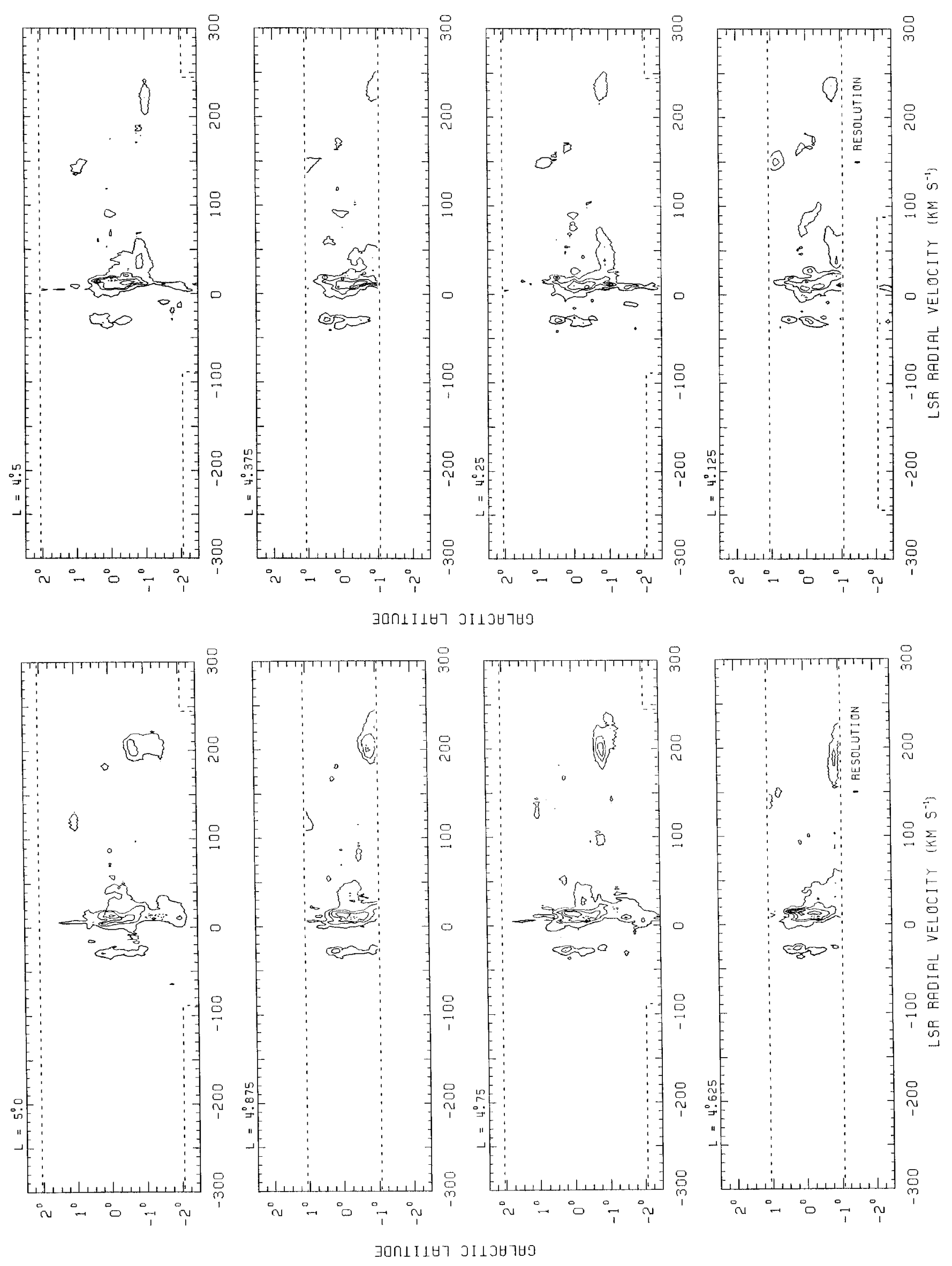

Fig. 4. continued 


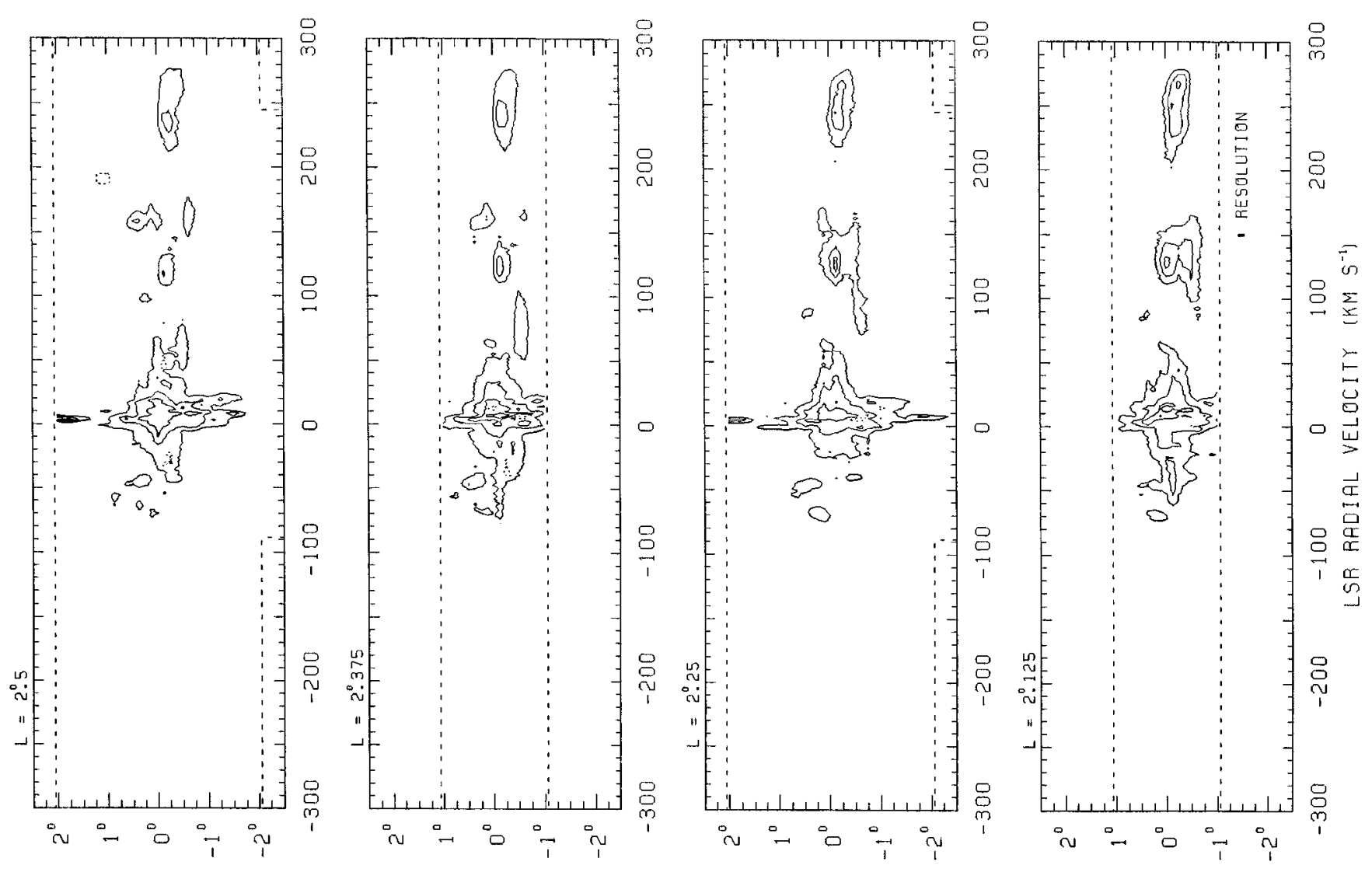

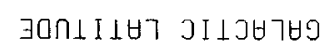

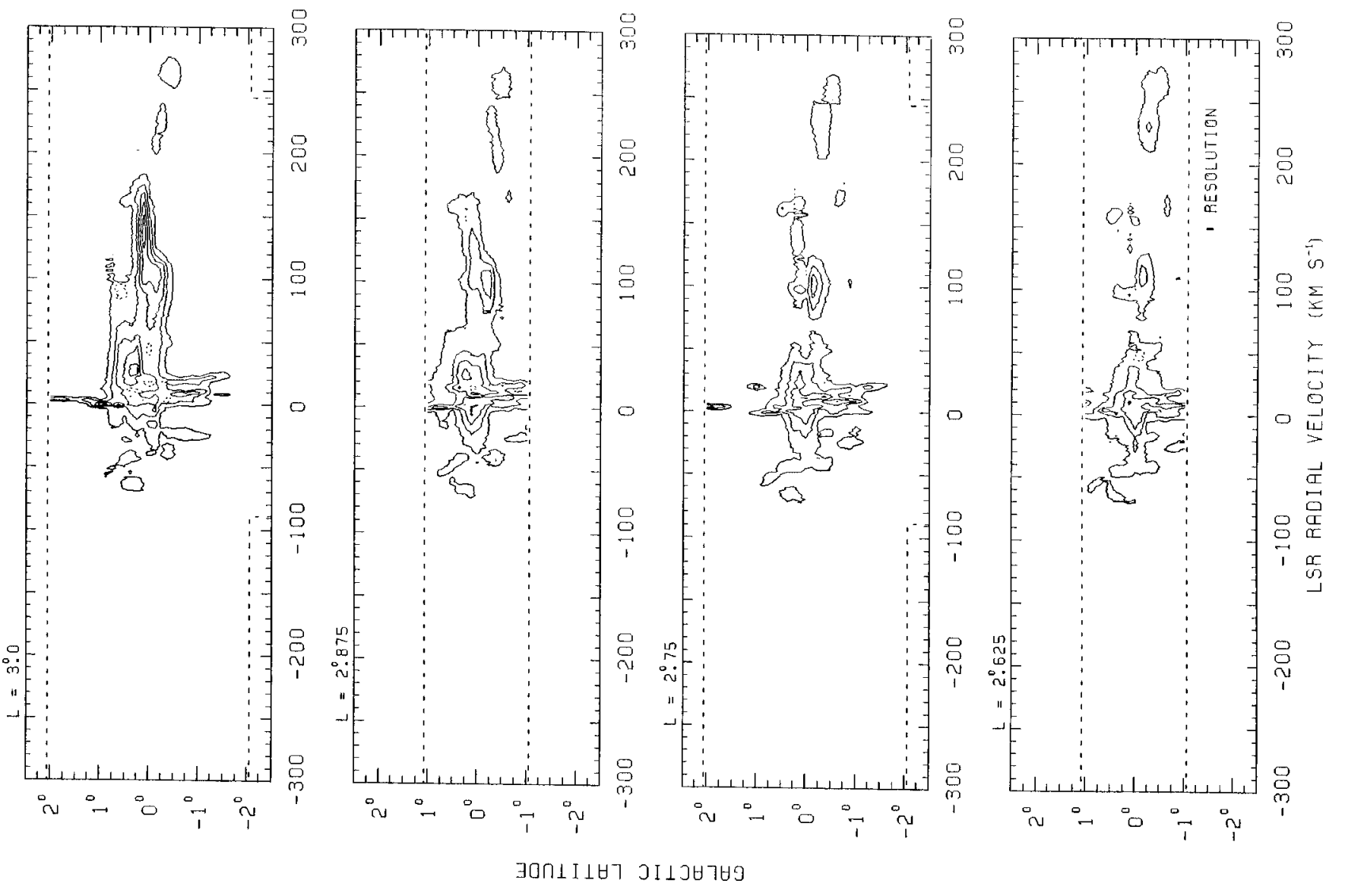

Fig. 4. continued 


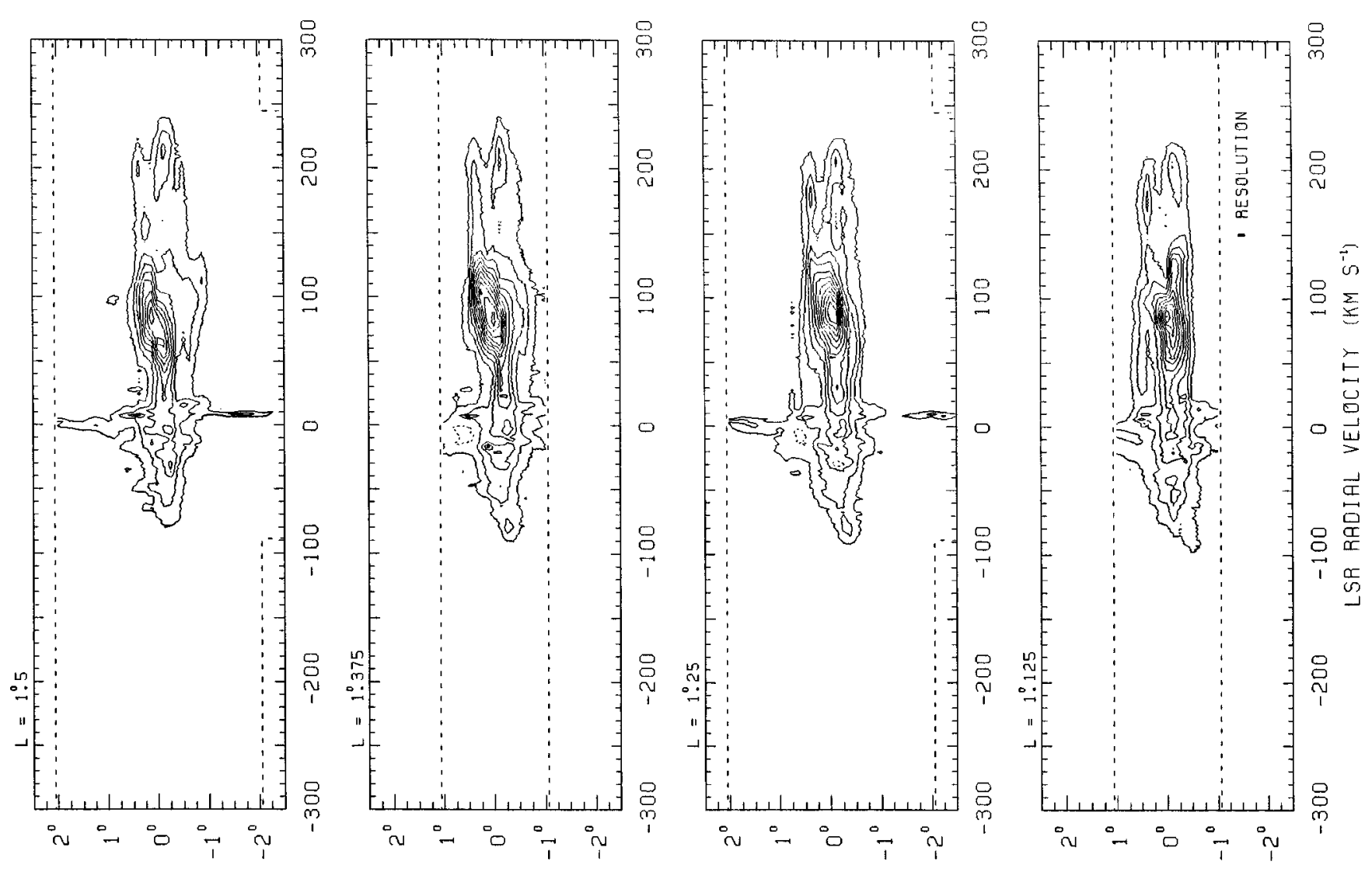

$\exists \square ก \perp I \perp \forall\urcorner ว I \perp J \forall\urcorner \forall \supset$

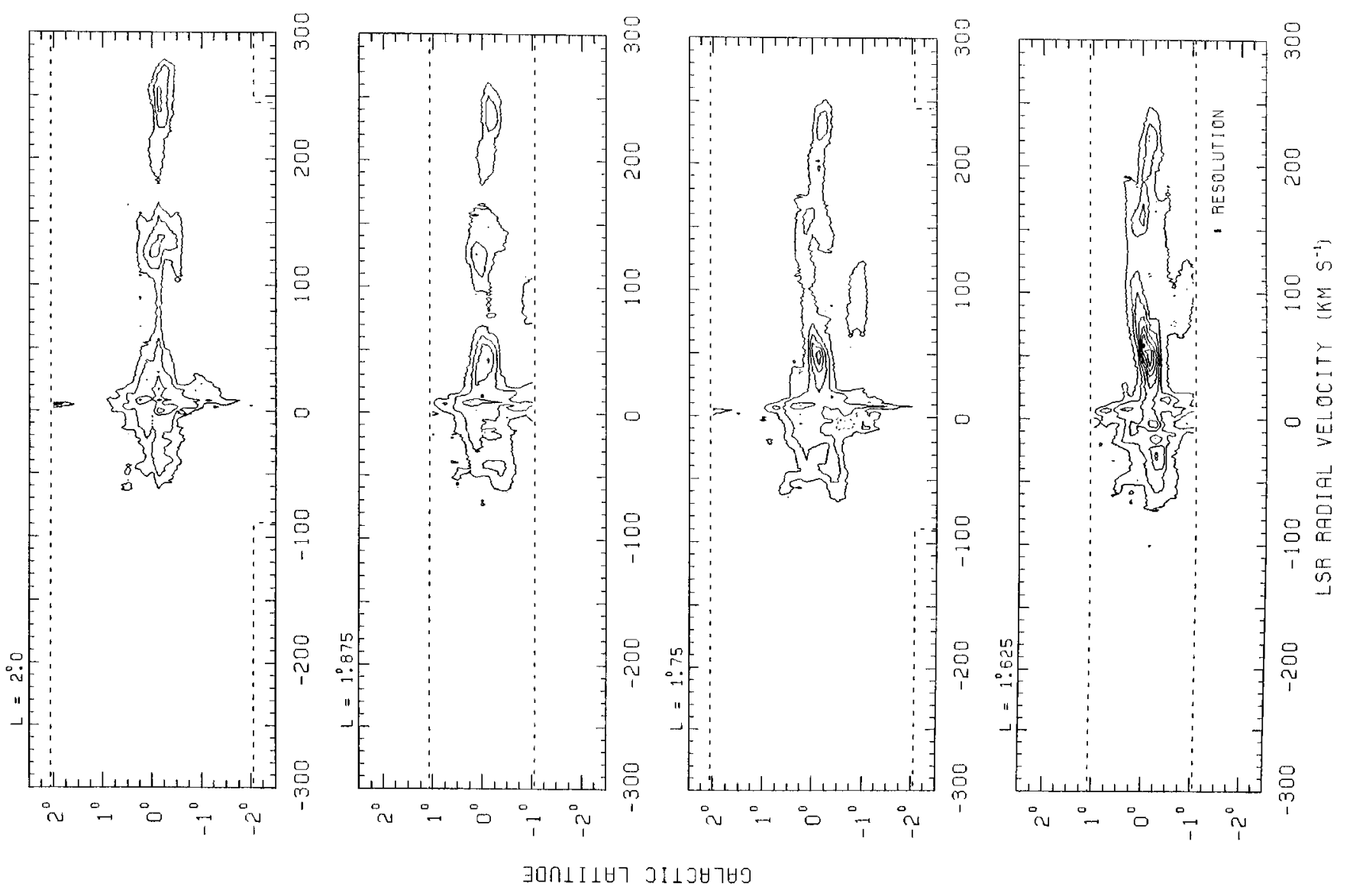

Fig. 4. continued 

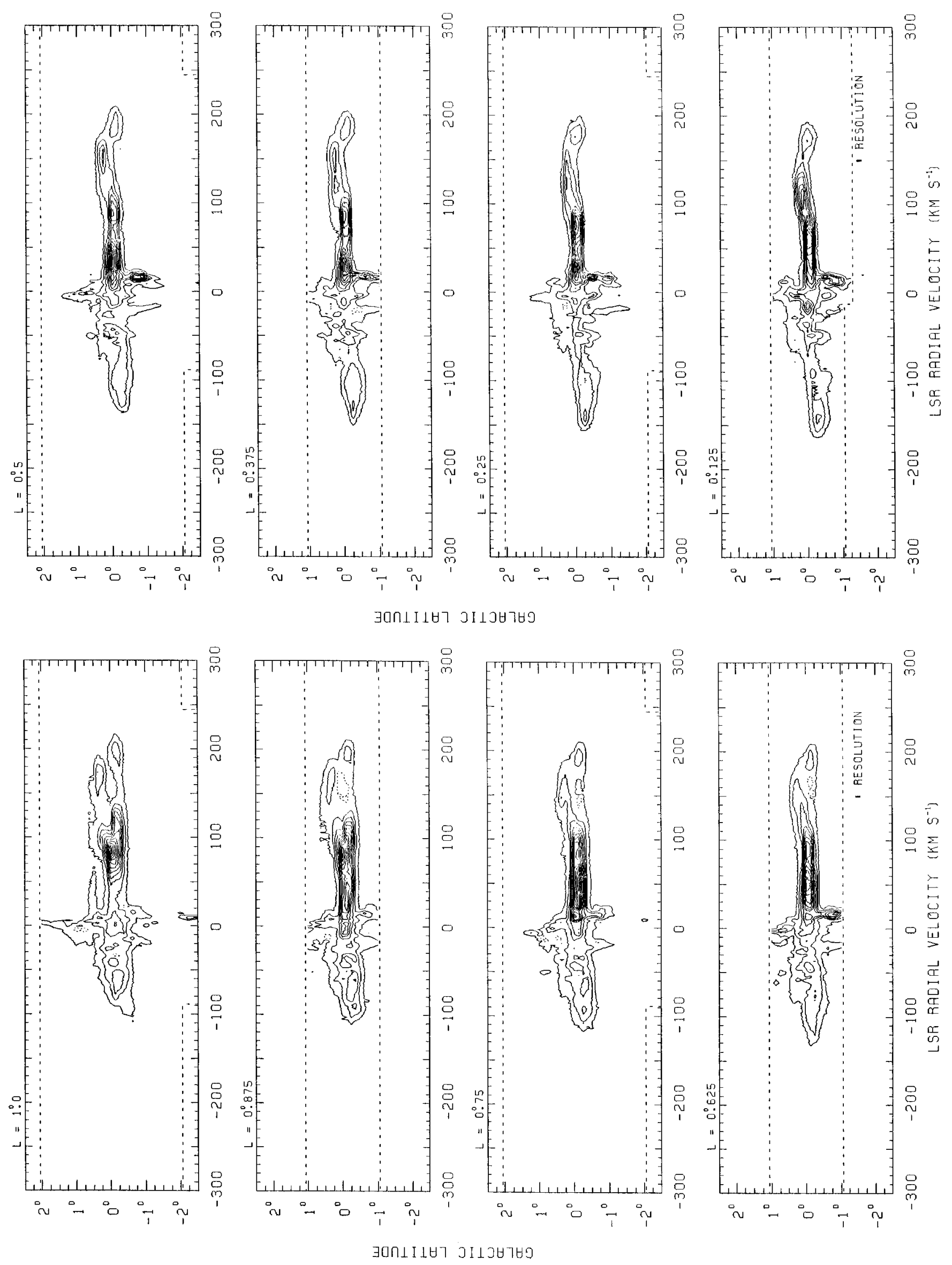

Fig. 4. continued 

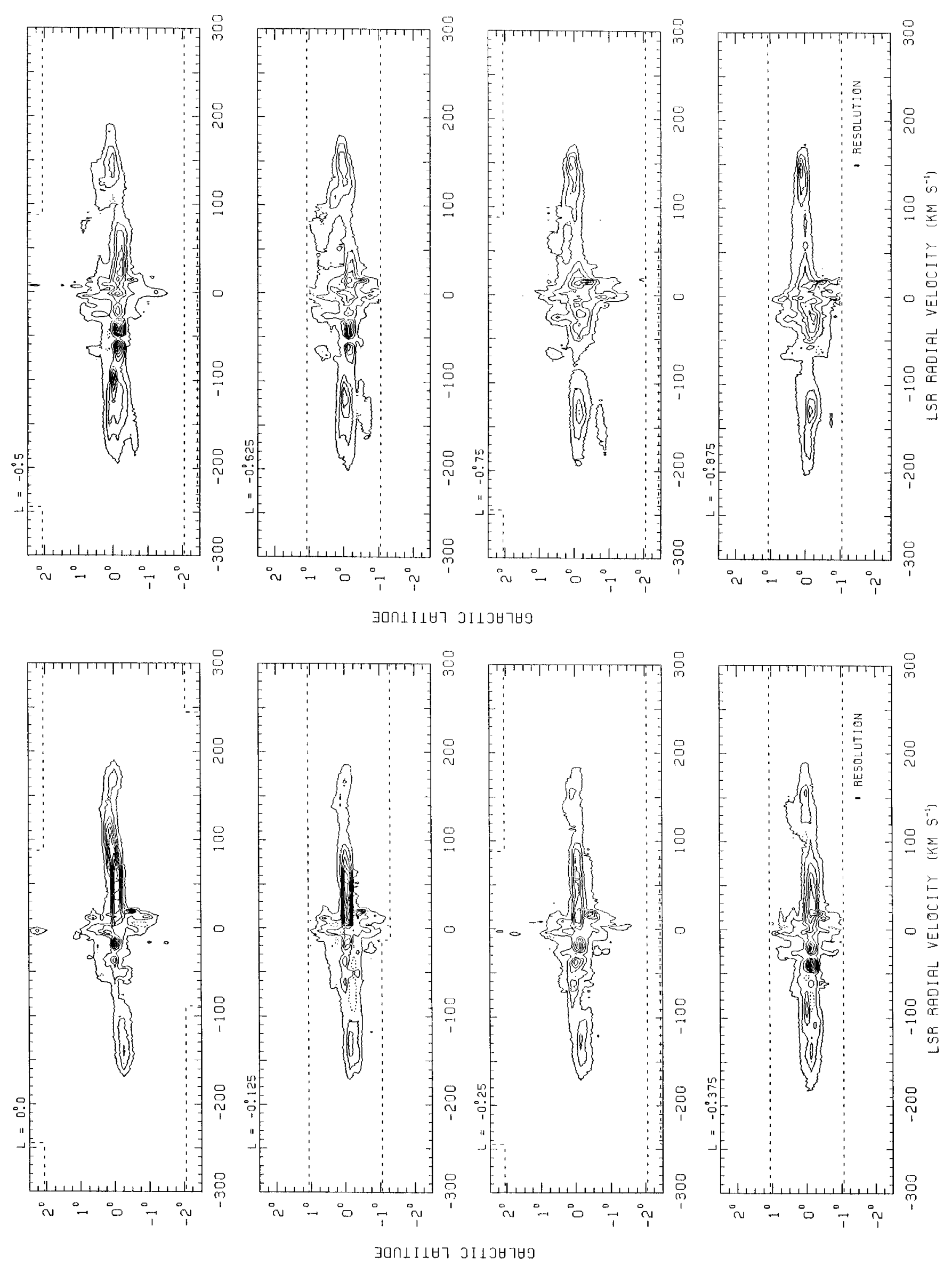

Fig. 4. continued 

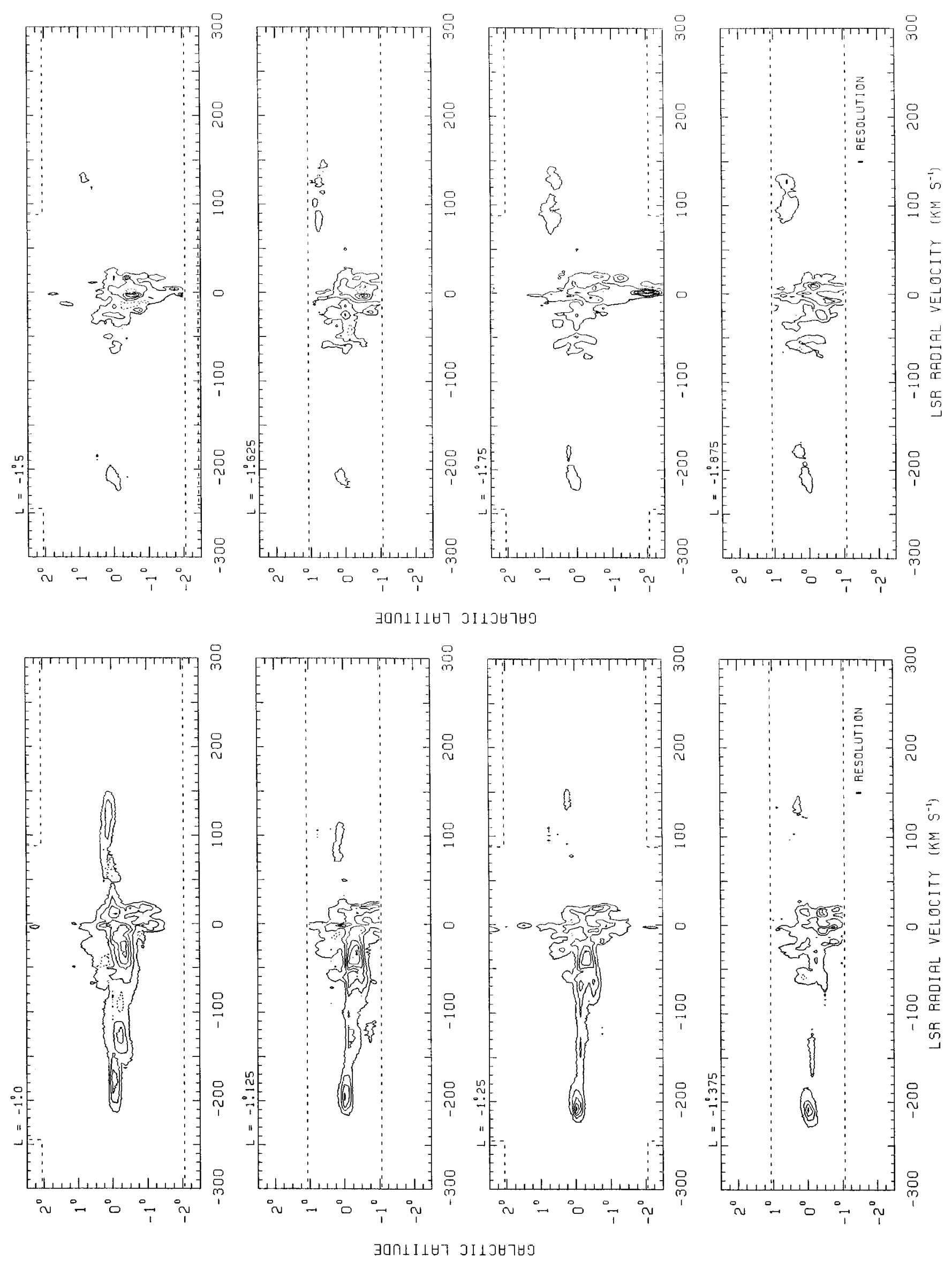

Fig. 4. continued 

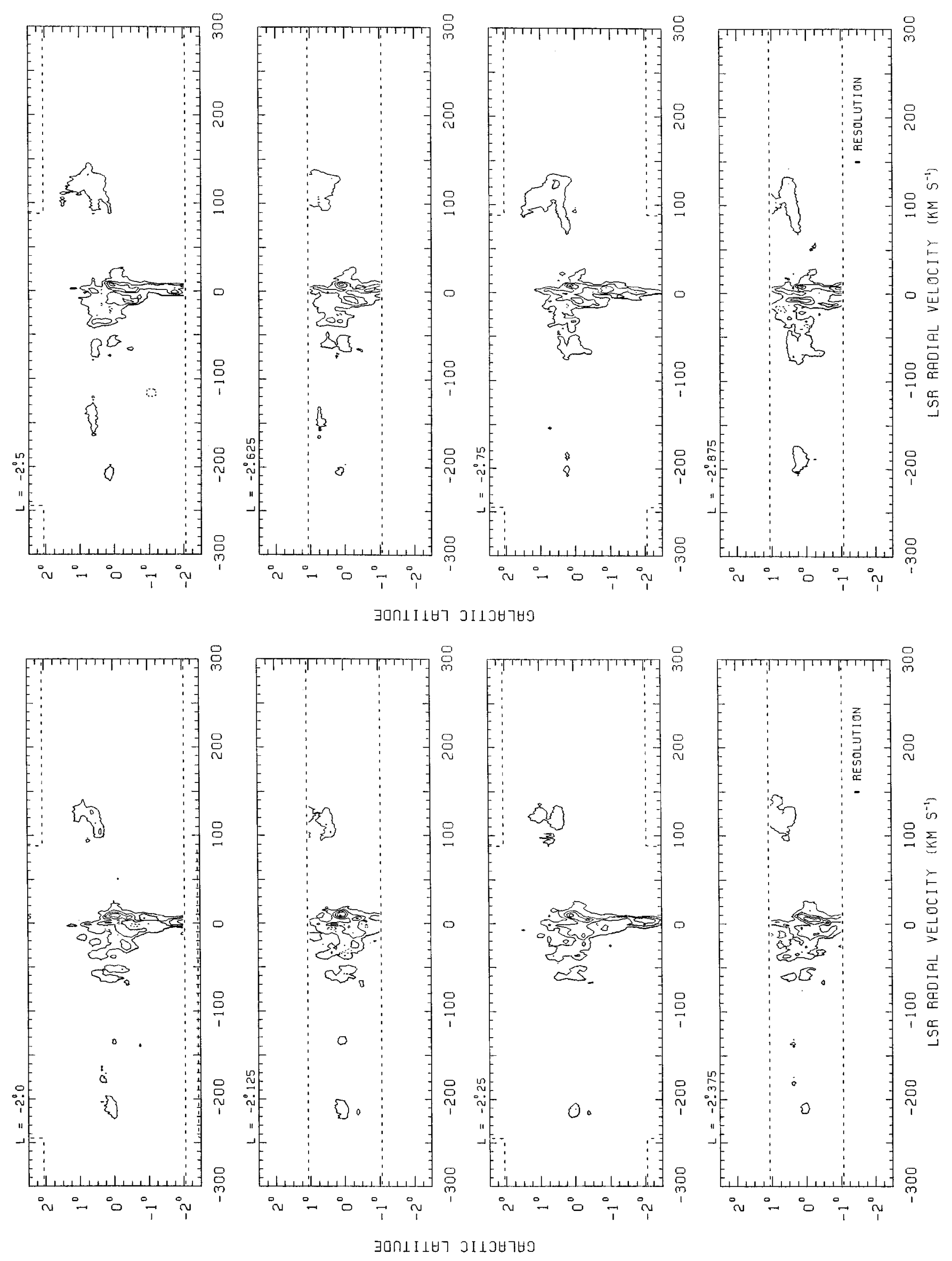

Fig. 4. continued 

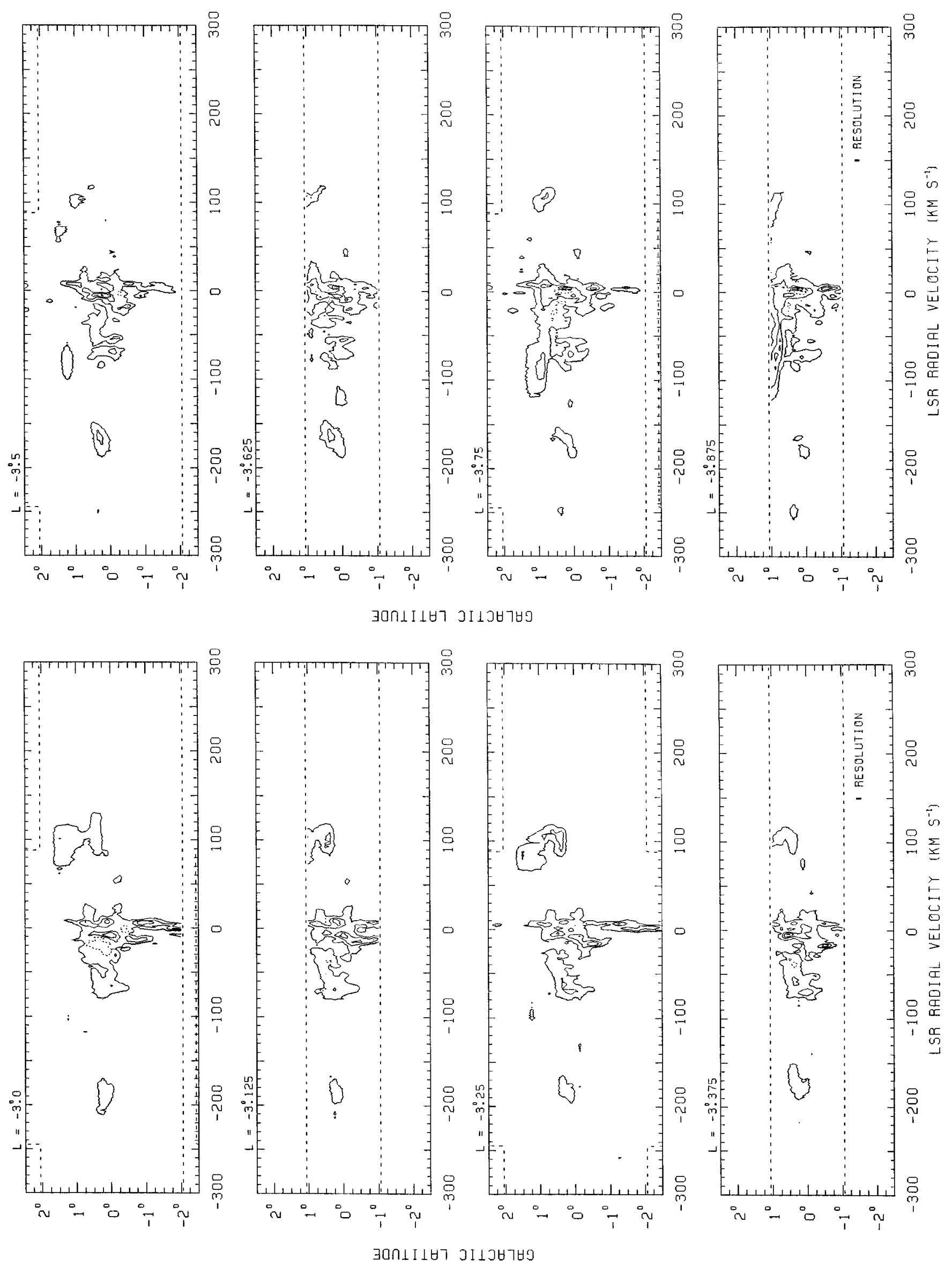

Fig. 4. continued 


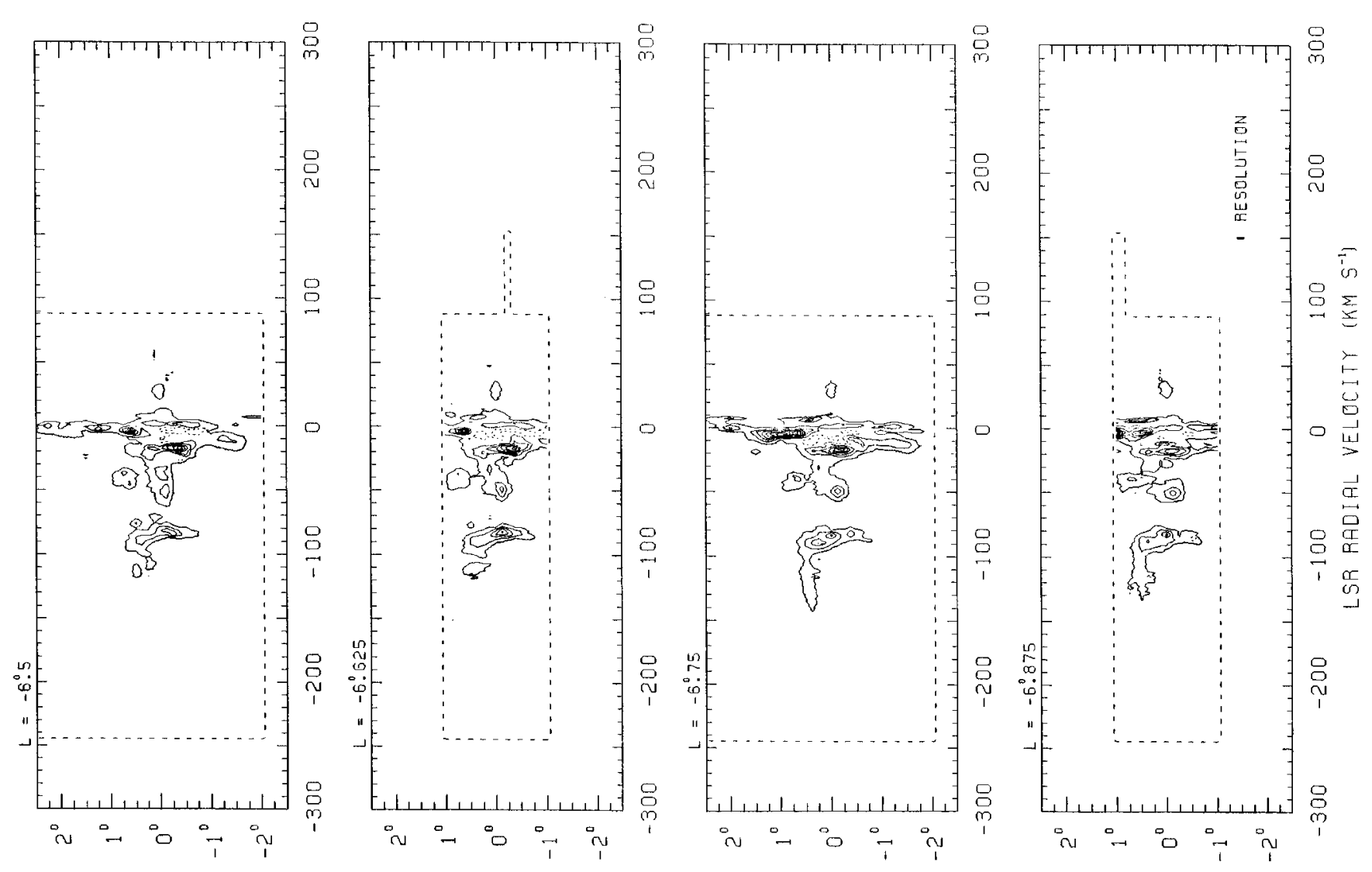

$\exists 0 \cap \perp \perp \forall\urcorner$ งI
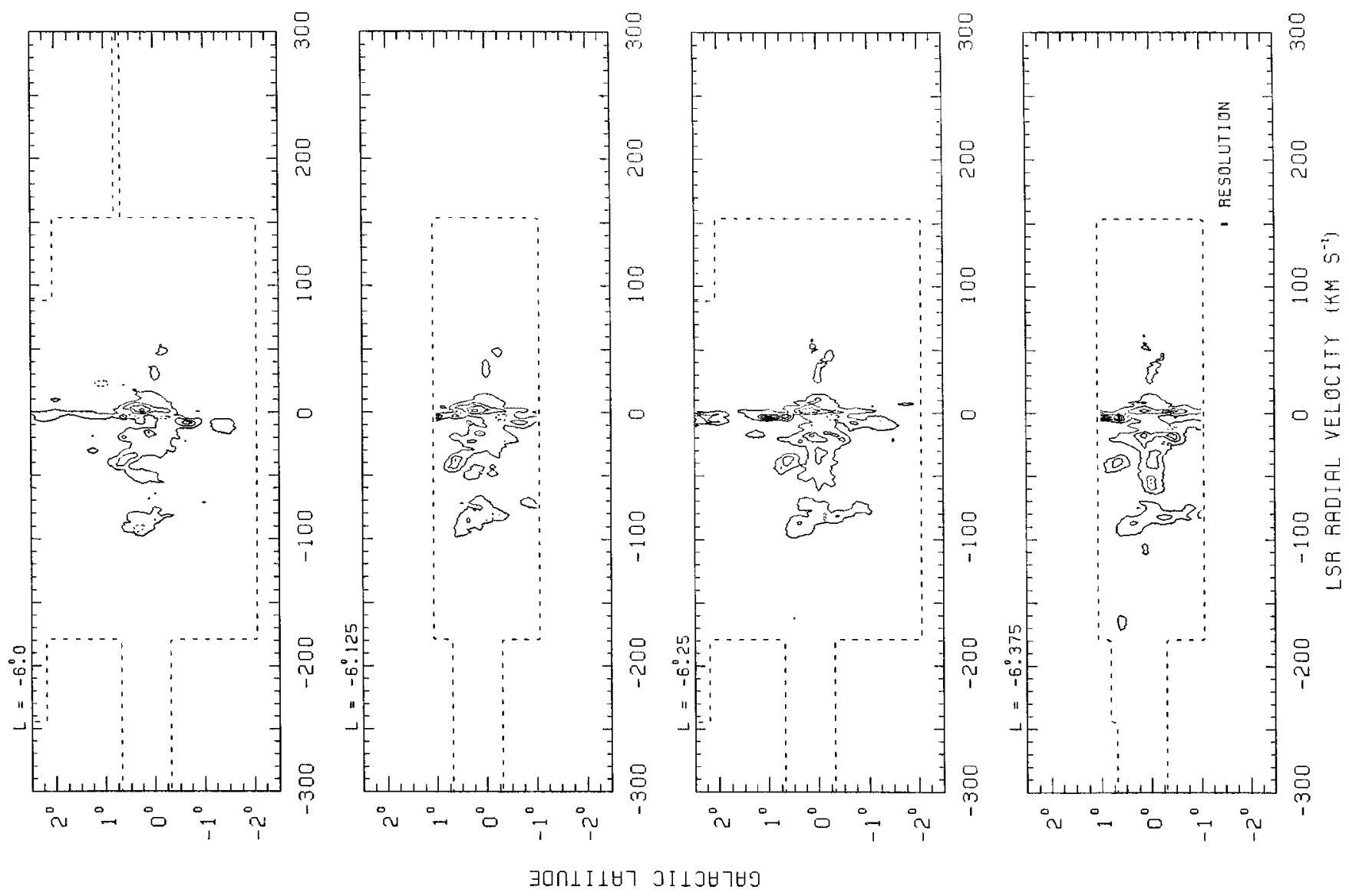

Fig. 4. continued 

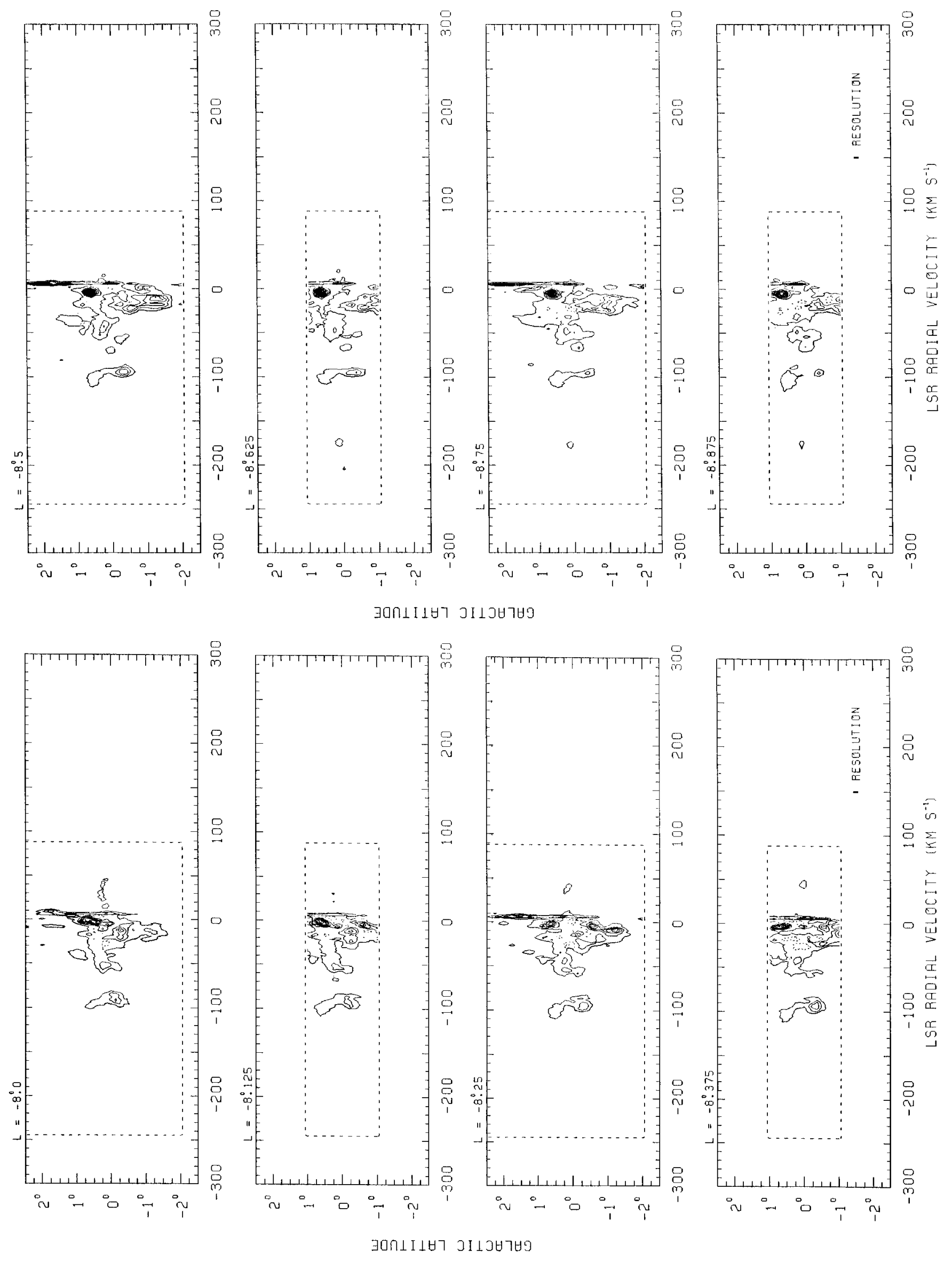

Fig. 4. continued 


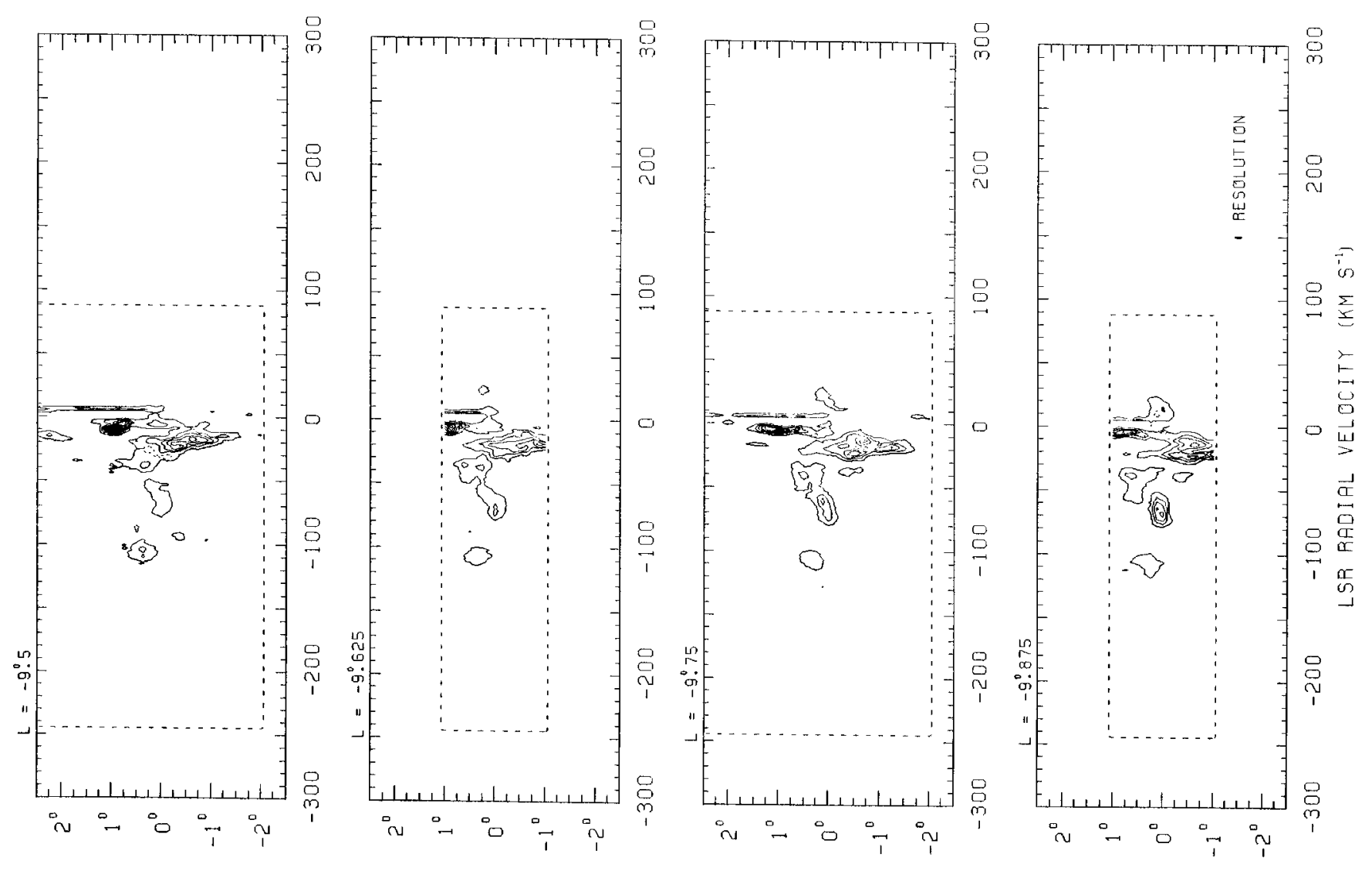

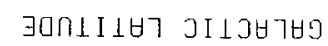

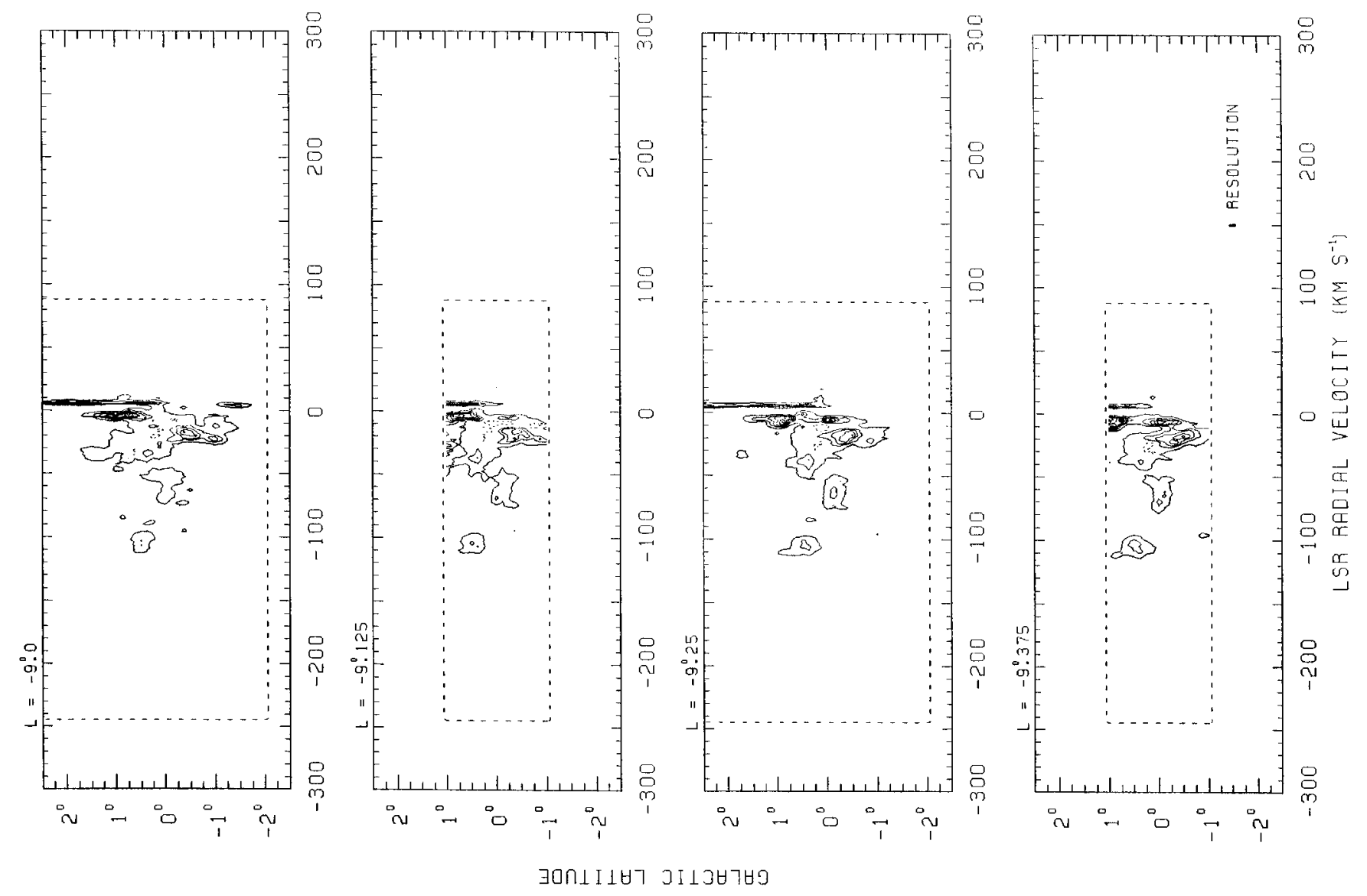

Fig. 4. continued 

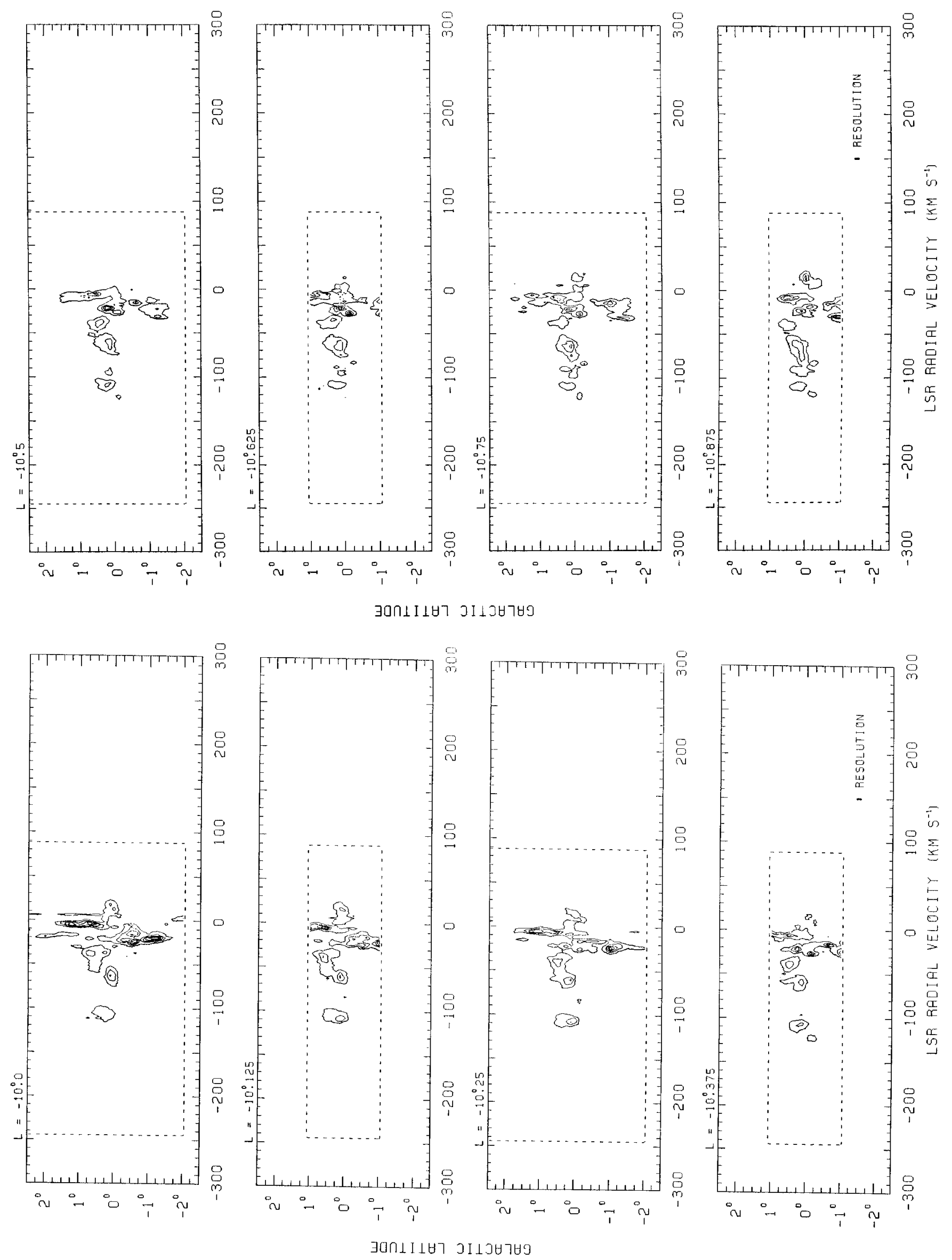

Fig. 4. continued 
